\title{
Dynamic remodeling of presynaptic endoplasmic reticulum is coordinated through actin and microtubule crosstalk and contributes to defective stimulus-response in Spinal Muscular Atrophy
}

\section{Michael Sendtner ( $\square$ Sendtner_M@ukw.de)}

University of Würzburg: Julius-Maximilians-Universitat Wurzburg https://orcid.org/0000-0002-47372974

\section{Chunchu Deng}

University of Würzburg: Julius-Maximilians-Universitat Wurzburg

Mehri Moradi

University of Würzburg: Julius-Maximilians-Universitat Wurzburg

\section{Sebastian Reinhard}

University of Würzburg: Julius-Maximilians-Universitat Wurzburg

\section{Sören Doose}

University of Würzburg: Julius-Maximilians-Universitat Wurzburg

Luisa Hennlein

University of Würzburg: Julius-Maximilians-Universitat Wurzburg

Sibylle Jablonka

University of Würzburg: Julius-Maximilians-Universitat Wurzburg

Markus Sauer

University of Würzburg: Julius-Maximilians-Universitat Wurzburg

\section{Research article}

Keywords: Spinal Muscular Atrophy, Presynaptic ER dynamics, BDNF stimulation, Local translation

Posted Date: March 29th, 2021

DOl: https://doi.org/10.21203/rs.3.rs-316151/v1

License: (1) This work is licensed under a Creative Commons Attribution 4.0 International License.

Read Full License 
Version of Record: A version of this preprint was published at Translational Neurodegeneration on June 2nd, 2022. See the published version at https://doi.org/10.1186/s40035-022-00304-2. 


\section{Abstract}

Background Axonal degeneration and defects in neuromuscular neurotransmission represent a pathological hallmark in spinal muscular atrophy (SMA) and other forms of motoneuron disease. These pathological changes do not only base on altered axonal and presynaptic architecture, but also on alterations in dynamic movements of organelles and subcellular structures that are not necessarily reflected by static histopathological changes. In neurons, a highly dynamic endoplasmic reticulum (ER) network exists in the axonal and presynaptic compartment which regulates $\mathrm{Ca} 2+$ homeostasis and synapse maintenance. However, the mechanisms of its dynamic regulation and mechanisms of dysfunction that contribute to neurodegeneration remain elusive. Methods Using high resolution microscopy and life imaging of cultured motoneurons from wildtype and a mouse model of spinal muscular atrophy, we investigated the dynamics of the axonal endoplasmic reticulum and ribosome distribution and activation. Results These studies revealed that the dynamic remodeling of ER in axonal filopodia of cultured motoneurons depends mainly on actin cytoskeleton. In Smn -deficient motoneurons, movements of ER in filopodia seems to be more affected than in the growth cone core. In addition, ribosome assembly that happens within seconds after exposure to Brain derived neurotrophic factors (BDNF) is reduced in axon terminals of Smn -deficient motoneurons, and also the association with ER as a response to extracellular stimuli is highly disturbed. Conclusions These findings do not only define a novel function of presynaptic ER in dynamic regulation of local translation. They also implicate impaired dynamic movements of axonal and presynaptic ER as a contributor to the pathophysiology of SMA and possibly also other neurodegenerative diseases.

\section{Background}

In neurons, the endoplasmic reticulum (ER) provides a continuous luminal space throughout the cytoplasm which extends into dendrites and axons $[1,2]$. Within presynaptic terminals, the ER forms a tubular network close to the active zone which is highly dynamic and undergoes constant movement and reorganization and regularly forms contact sites with the plasma membrane [1]. The dynamic movements of ER are regulated by the cytoskeleton and motor proteins. Live-cell fluorescence microscopy studies have shown that microtubules regulate ER movements in animal cells [3], whereas in plants [4] and budding yeast cells [5] ER movements require actin. Similarly, in neurons, microtubules provide a structural backbone for gross dendritic and axonal ER movements [6]. Other studies revealed a specific role of myosin Va in ER transport along actin filaments into dendritic spines, demonstrating that ER import into these subcellular compartments might be actin dependent [7]. Whether similar actin dependent mechanisms also determine the movement of axonal ER remained unclear. In this study, we show that in axons, the ER appears associated with actin filaments, especially in growth cone filopodia where actin filaments are highly enriched. The importance of the axonal ER dynamic regulation is highlighted by mutations in ER-shaping or ER-receptor proteins which impair ER remodeling and associate with neurodegenerative diseases such as amyotrophic lateral sclerosis (ALS) [8] or spastic paraplegia (HSP) $[9,10]$. 
In neurons, rough cisternal ER (RER), which is the major site for protein synthesis, folding, processing and secretion is mainly restricted to the somatodendritic compartment [11-15]. Axons are traditionally considered to be devoid of RER, as demonstrated by ultrastructure EM and thought to exhibit only smooth ER $[16,17]$. The function of such dendritic and axonal ER has been suggested to be limited to lipid metabolism, $\mathrm{Ca}^{2+}$ homeostasis, and to function in contacting membranous organelles to regulate their biogenesis and maintenance $[1,6,12,17]$. Nevertheless, despite emerging evidence of intra-axonal translation of mRNAs encoding membrane and secreted proteins in axons of isolated neurons, there is no direct evidence for the existence of a rough ER in axons that could process such locally synthesized proteins for integration into the axoplasmic membrane and secretion [18].

Here, we investigated the interaction of ribosomes with ER in the presynaptic terminals of cultured motoneurons and found that ribosomes undergo rapid changes in distribution and structure in response to extracellular cues. In particular, they relocate to the ER where they could accomplish local translation of membrane-associated and secreted proteins. Furthermore, we unraveled the underlying mechanisms of regulation of presynaptic ER dynamic movements and show that fast dynamic elongation of ER into axonal filopodia is regulated mainly by actin, whereas slow ER movement in the growth cone core requires a coordinated actin and microtubule cytoskeleton. Thus, we provide evidence of a novel function of axonal ER in local protein synthesis in addition to its previously described roles.

This observation also seems to be relevant for the pathophysiology of spinal muscular atrophy (SMA), an autosomal recessive motoneuron disease caused by loss of SMN protein [19]. Isolated motoneurons from SMA mouse models show severe defects in mRNA localization and intra-axonal protein synthesis $[20,21]$. Our data implicate that ER dynamic movements are also impaired in Smn-deficient motoneurons. In particular, the rapid response of ribosomes to extracellular stimuli is not accomplished. Thus, in addition to mRNA mislocalization, aberrant ER dynamics and ribosome response to extracellular signaling could affect axonal growth and presynaptic function and maintenance, thereby contributing to the pathology of SMA.

\section{Materials And Methods}

\section{Animals}

The SMA mouse model is a transgenic mouse expressing two copies of the human SMN2 gene, mated onto a mouse null Smn-/- background, resulting in a severe phenotype resembling type I SMA in humans [82]. TrkB knockout mice [83] were obtained from the University of California, Davis (MMRRC: 000188, B6; 129S4-Ntrk2 < tm1Rohr >) and maintained on a C57BI/6 background. CD1 mice were used for motoneuron cell cultures from WT mice. All mice were housed in the animal facility of the Institute of Clinical Neurobiology, University Hospital of Wuerzburg. All mouse procedures were performed according to the regulations on animal protection of the German federal law and of the Association for Assessment and Accreditation of Laboratory Animal care, approved by the local authorities. 


\section{Culture of embryonic mouse motoneurons}

Embryonic mouse motoneuron culture from the lumbar spinal cord was performed as previously described [84]. Lumbar spinal cords were dissected from E13.5 mouse embryos, digested with trypsin (Worthington), triturated and then transferred onto a panning plate coated with anti-p75 antibody (MLR2, Abcam) for enrichment of motoneurons. For lentiviral transduction, $\mathrm{pSIH}$-mCherry-KDEL construct expressing lentiviruses were added to the suspension of motoneurons before plating on polyornithine(PORN) and human merosin-coated (CC085, Merck-Millipore) plates. Motoneurons were cultured in NB medium supplemented with $500 \mu \mathrm{M}$ Glutamax, 2\% heat-inactivated horse serum (Gibco), 2\% B27 (Thermo Fisher Scientific) and $5 \mathrm{ng} / \mathrm{ml} \mathrm{BDNF}$ for 5 to 6 days in a humidified CO2 incubator at $37^{\circ} \mathrm{C}$. Medium was changed $24 \mathrm{~h}$ after plating and then every other day. Merosin consists of Laminin211 ( $a 2 \beta 1 \gamma 1)$, enriched in extrasynaptic basal lamina and Laminin221 (a2ß2y1), which is specifically expressed at the cleft of NMJs and regulates formation, maturation and maintenance of NMJs $[85,86]$. Thus, culturing motoneurons on merosin induces the maturation of presynaptic structures in axon terminals. Compartmentalized motoneuron cultures were prepared as described previously [21]. To drive axonal growth into the axonal compartment, $20 \mathrm{ng} / \mathrm{ml} \mathrm{BDNF}$ and CNTF were added into the axonal compartments, while only $5 \mathrm{ng} / \mathrm{ml}$ CNTF was added into the somatodendritic compartments.

\section{Immunohistochemistry analysis of NMJs using transversus abdominis muscle (TVA)}

CD1 mouse was killed at P5. TVA was dissected in the extracellular physiological solution $(135 \mathrm{mM} \mathrm{NaCl}$, $12 \mathrm{mM} \mathrm{NaHCO} 3,5 \mathrm{mM} \mathrm{KCl}, 1 \mathrm{mM} \mathrm{MgCl} 2,2 \mathrm{mM} \mathrm{CaCl} 2,20 \mathrm{mM}$ Glucose). After fixation with 4\% PLP buffer ( $4 \% \mathrm{PFA})$ at $4^{\circ} \mathrm{C}$ for $90 \mathrm{~min}$, muscles were washed with $0.1 \mathrm{M}$ glycine on a shaker for $30 \mathrm{~min}$ and permeabilized with PBST (0.5\% Triton X-100) twice for $5 \mathrm{~min}$, twice for $10 \mathrm{~min}$ and twice for $30 \mathrm{~min}$. After permeabilization, TVA muscles were incubated with block solution including $5 \%$ BSA and $2 \%$ donkey serum in PBST at RT for $3 \mathrm{~h}$, and then with primary antibodies in block solution at $4^{\circ} \mathrm{C}$ for 2 nights on a shaker. Then, preparations were washed with PBST 3 x each 15 min and secondary antibodies were incubated at RT for $1 \mathrm{~h}$. After $3 \mathrm{x}$ wash with PBST, preparations were rinsed in water and embedded using Aqua-Poly/Mount (18606-20, Polysciences). The following primary and secondary antibodies were used: polyclonal chicken anti-calreticulin (PA1-902A, Thermo Fisher Scientific; 1:500), monoclonal mouse antirRNA (Y10B) (MA116628, Thermo Fisher Scientific; 1:500), polyclonal guinea pig anti-synaptophysin 1 (101004, Synaptic Systems; 1:1000), donkey anti-chicken IgY H+L (Alexa Fluor488, 703-545-155, Jackson ImmunoResearch; 1:500), goat anti-mouse IgG (H+L) (115-165-146, Jackson ImmunoResearch; 1:500) and donkey anti-guinea pig IgG (H+L) AffiniPure (706-175-148, Jackson ImmunoResearch; 1:500).

\section{Cloning and lentivirus production}

For cloning of pSIH-mCherry-KDEL, mCherry was first amplified by PCR using a commercially available plasmid as template (primers: forward: 5'-ACTCGTCGACGTGAGCAAGGGCGAGGAGGAT-3'; reverse: 5'GAATGCGGCCGCCTTGTACAGCTCGTCCATGCC-3'), and cut by Sall and Notl enzymes, followed by insertion into the pCMV-ECS2-CMV-myc-ER vector backbone containing a KDEL sequence. The 
assembled mCherry-KDEL fragment was amplified by PCR from the above vector (primers: forward: 5'tttgacctccatagaagattCCACCATGGGATGGAGCTG-3, reverse: 5'-

tgtaatccagaggttgattgCTACAGCTCGTCCTTCTCG-3'), and the product was inserted into a lentiviral expression vector ( $\mathrm{pSIH}-\mathrm{H})$ containing CMV promotor using NEBbuilder HIFI kit (NEW ENGLAND Biolabs). To co-express GFP-actin and mCherry-KDEL, a previously described GFP-actin construct was used [87]. Both mCherry-KDEL and GFP-actin were first amplified by PCR and purified fragments were inserted into a pSIH-CMV-IRES vector. Lentiviruses were produced in HEK ${ }^{293 T}$ cells using pCMV-VSVG and pCMVAR8.91 helper plasmids [88]. HEK ${ }^{293 T}$ cells were transfected with calcium-phosphate reagents, and viral supernatants were harvested after $47 \mathrm{~h}$ by ultracentrifugation. NSC34 cells were used for virus titer test.

\section{BDNF stimulation and immunocytochemistry of cultured motoneurons}

At DIV6, motoneurons were fixed with $4 \%$ PFA for $10 \mathrm{~min}$ at RT and permeabilized with $0.1 \%$ or $0.3 \%$ Triton X-100 for 10 min, followed by $3 \mathrm{x}$ wash with PBS. After incubation with block solution (10\% donkey serum and $2 \%$ BSA in PBS) at RT for $1 \mathrm{~h}$, primary antibodies were added and incubated at $4^{\circ} \mathrm{C}$ overnight. On the second day, motoneurons were washed trice with PBS and incubated with secondary antibodies for $1 \mathrm{~h}$, followed by another $3 \mathrm{x}$ wash. Aqua Poly/Mount (18606-20, Polysciences) was used for embedding. For $\beta$-actin staining, motoneurons were permeabilized with ice-cold methanol for $5 \mathrm{~min}$ at -20 ${ }^{\circ} \mathrm{C}$. For the BDNF stimulation experiments, motoneurons were first cultured with $5 \mathrm{ng} / \mathrm{ml}$ BDNF. At DIV5, medium was completely aspired and cells were washed twice with NB medium to completely remove BDNF from all the surfaces. Cells were maintained in NB medium supplemented with $2 \% \mathrm{HS}$ and $2 \%$ B27 overnight in the absence of neurotrophic factors. On the next day, BDNF stimulation was conducted by directly adding $40 \mathrm{ng} / \mathrm{ml} \mathrm{BDNF}$ into the cell culture medium and cultures were kept on a $37^{\circ} \mathrm{C}$ hotplate. For different stimulation times, BDNF containing medium was removed after $10 \mathrm{sec}, 1 \mathrm{~min}$ and $10 \mathrm{~min}$ stimulation and 4\% PFA was directly added onto cells. For the no BDNF control group, the same amount of NB medium without BDNF was added. The following primary antibodies were used: monoclonal mouse anti-a-Tubulin (T5168, Sigma-Aldrich; 1:1000), polyclonal goat anti-TrkB (AF1494, Bio-Techne Sales Corp; 1:500), monoclonal mouse anti-rRNA (Y10b) (MA116628, Thermo Fisher Scientific; 1:500), polyclonal goat anti-ribosomal protein L8 (SAB2500882, Sigma-Aldrich; 1:500), monoclonal mouse antibeta Actin (GTX26276, GeneTex; 1:1000), polyclonal rabbit anti-Tau (T6402,Sigma-Aldrich;1:1000), polyclonal rabbit anti-eEF2 (23325, Cell Signaling Technology; 1: 50), monoclonal mouse anti-S6 ribosomal protein (MA515123, Thermo Fisher Scientific; 1:500), polyclonal rabbit anti-RPL24 (PA530157, Thermo Fisher Scientific; 1:100), polyclonal guinea pig antiserum RFP (390004, Synaptic Systems; 1:500) and monoclonal rat anti-mCherry (M11217, Thermo Fisher Scientific; 1:1500). Secondary antibodies: donkey anti-mouse IgG (H+L) (Alexa Fluor 488; A21202,Life Technologies;1:500), donkey anti-rabbit IgG $(\mathrm{H}+\mathrm{l})$ AffiniPure (Alexa Fluor 488; 711-545-152, Jackson ImmunoResearch; 1:500), donkey anti-guinea pig IgG (H+L) AffiniPure (Cy3; 706-165-148: Jackson ImmunoResearch; 1:500), donkey anti-rat IgG $(\mathrm{H}+\mathrm{L})$ AffiniPure (Cy3; 712-165-150; Jackson ImmunoResearch), donkey anti-rabbit IgG (H+L) AffiniPure (Cy3; 711-165-152; Jackson ImmunoResearch), donkey anti-goat IgG (H+L) AffiniPure (Cy3;705-165-147: Jackson ImmunoResearch), donkey anti-mouse IgG (H+L) (Cy3; 715-165-151; Jackson ImmunoResearch; 
1:500), donkey anti-goat IgG (H+L) AffiniPure (Alexa Fluor 647; 705-605-003; Jackson ImmunoResearch), donkey anti-mouse IgG $(\mathrm{H}+\mathrm{L})$ highly cross-adsorbed (Alexa Fluor 647; A31571; Invitrogen), donkey antirabbit IgG (H+L) AffiniPure (Cy5; 711-175-152; Jackson ImmunoResearch). F-actin was labeled with Alexa Fluor647-conjugated Phalloidin (A22287; Invitrogen). All secondary antibodies were diluted 1:500 in TBST.

\section{Puromycin experiments}

Motoneurons were incubated with $10 \mu \mathrm{g} / \mathrm{ml}$ puromycin for $10 \mathrm{~min}$ and BDNF stimulation was carried out as described in the above section. Cells were then fixed and immunostained using anti-puromycin immunostaining. Nocodazole was used to disrupt microtubule-dependent axonal transport and anisomycin was used as translational inhibitor. Cells were treated with $10 \mu \mathrm{M}$ nocodazole for $2 \mathrm{~h}$ and 100 $\mathrm{ng} / \mathrm{ml}$ anisomycin for $1 \mathrm{~h}$ prior to as well as during puromycin incubation followed by BDNF stimulation. Primary and secondary antibodies used were: monoclonal mouse anti-puromycin (clone 12D10, MABE343, Merck Millipore; 1:1000), polyclonal rabbit anti-Tau (T6402, Sigma-Aldrich; 1:1000), donkey anti-mouse IgG $(\mathrm{H}+\mathrm{L})$ (Alexa Fluor 488; A21202, Life Technologies; 1:500), and donkey anti-rabbit IgG $(\mathrm{H}+\mathrm{L})$ AffiniPure (Cy3; 711-165-152; Jackson ImmunoResearch).

\section{Image acquisition and data analysis}

Image acquisition was done with a standard Olympus Fluoview 1000 confocal system with a 60x NA 1.35 oil objective. Structured illumination microscopy (SIM) imaging was performed on a commercial ELYRA S. 1 microscope (Zeiss AG). The setup is equipped with a Plan-Apochromat 63x/1.40 immersion-oil based objective and four excitation lasers, a $405 \mathrm{~nm}$ diode (50 mW), a $488 \mathrm{~nm}$ OPSL (100 mW), a $561 \mathrm{~nm}$ OPSL (100 mW) and a $642 \mathrm{~nm}$ diode laser $(150 \mathrm{~mW})$. For quantification of immunofluorescence signals, mean gray values of images were measured from unprocessed raw data after background subtraction using ImageJ-win64. For quantification of SIM data represented in Fig. 4, 6 and 9, co-clusters of RPL24/RPS6, Y10B/eEF2 as well as RPL24/RPS6/ER and Y10B/eEF2/ER were counted manually. For this, maximum projections of single SIM channels (RPL24, RPS6 and mCherry-ER or Y10B, eEF2 and mCherry-ER channels) were first created using ImageJ. An automatic linear adjustment of contrast and brightness was applied to the whole z-projection image of each single channel and these were merged into a RGB image. Co-clusters were defined as overlapping dots from RPL24, RPS6 and mCherry-ER channels or Y10B, eEF2 and mCherry-ER channels on the RGB image which had a diameter of more than $350 \mathrm{~nm}$. GraphPad Prism 8 software was used for all statistical analyses. Data are shown in scatter dot plots with mean \pm SEM, unless otherwise mentioned. For a better visibility, linear contrast enhancement was applied to all representative images using Adobe Photoshop.

\section{Live cell Imaging and data quantification}

Approximately, 40,000 motoneurons were transduced with lentivirus expressing pSIH-mCherry-KDEL or pSIH-GFP-actin-IRES-mCherry-KDEL and cultured on PORN/ merosin-coated $35 \mathrm{~mm}$ high $\mu$-dishes (81156, IBIDI) for 6 days. For live cell imaging, an inverted epifluorescence microscopy (TE2000; Nikon) was used 
that was equipped with a perfect focus system, heated stage chamber (TOKAI HIT CO, LTD) at $37^{\circ} \mathrm{C}, 5 \%$ CO2 and 60x 1.4 NA objective. Time series were captured at a speed of $2 \mathrm{sec}$ per frame over $15 \mathrm{~min}$. 12bit images of $1.024 \times 1.024$ pixels were acquired with an Orca Flash 4.0 V2 camera (Hamamatsu Photonics), controlled by Nikon Element image software. For quantification of ER dynamics, Image Correlation Spectroscopy (ICS) [25] was implemented in python. This approach was used as described previously by Wiseman et,. al. Briefly, molecular movements of ER or molecular transport of other organelles are determined based on flow or diffusion in an image time series, with $\mathrm{N}, \mathrm{M}, \mathrm{T}$ indicating the two dimensions in space and the time dimension respectively. denote the corresponding running indices the basic workflow starts by defining a space-time window, i.e., a subspace of over $\Delta t=10$ consecutive frames. This subspace is rasterized over the image with a sampling rate of $\Delta \mathrm{i}=\Delta \mathrm{j}=4$. For each of those samples a correlation window is computed, describing the overlap of signal for a given shift per time frame. The maximum of this spectrum is the point of maximum correlation and indicates a shift of signal from one image to this next, by . To achieve sub-pixel accuracy a 2D Gaussian function is fitted to the correlation spectrum using the Levenberg-Marquardt-Algorithm. The initial parameters were chosen as amplitude, center coordinates in , , center coordinates in , , standard deviation in , , standard deviation in , . An additional parameter rotating the coordinate system by was initialized as 0 . The fit results for and indicate a directed shift of signal. A larger or indicates an overall lower correlation, i.e., the signal losing its shape. To compute overall dynamics, three distinct subspaces are classified: (i) subspaces with a mean intensity less than $10 \%$ of the maximum intensity are considered noise. (ii) Subspaces with a mean intensity that is larger than $40 \%$ of the maximum intensity are classified as core. (iii) Subspaces with an intensity in between is considered as filopodia. The overall dynamics value for a class is the mean dynamics value of all subspaces. Kymograph-analysis of ER dynamics was carried out using Image $\mathrm{J}$ as described previously [20]. Briefly, a Z-projection image was made from all the frames of a live cell image, followed by the creation of a multiple kymograph along a line drawn tracking the ER movements in filopodia or core. Distance changed per movement and frequency of movements over 15 min were calculated from multiple kymographs and plotted in a graph. Growth cones without detectable ER movements were classified as failure filopodia or core ER movement.

\section{Western blotting}

For Western blot analysis, approximately 300,000 motoneurons were plated onto a PORN/merosin-coated 24-well cell culture dish. At DIV6, BDNF-deprived neurons were stimulated with $40 \mathrm{ng} / \mathrm{ml} \mathrm{BDNF}$ for $10 \mathrm{sec}$, $30 \mathrm{sec}, 1 \mathrm{~min}$ and $10 \mathrm{~min}$ and cells were lysed directly in 2x Laemmli buffer (125 mM Tris, pH 6.8, 10\% SDS, $50 \%$ glycerol, $25 \% \beta$-mercaptoethanol and $0.2 \%$ bromophenol blue). The protein lysates were boiled at $99^{\circ} \mathrm{C}$ for $10 \mathrm{~min}$. Protein extracts were separated using $4-12 \%$ Gradient SDS-PAGE gels. Proteins were then blotted onto nitrocellulose membranes and primary antibodies were incubated on a shaker at $4^{\circ} \mathrm{C}$ overnight. Primary antibodies were washed with TBST and secondary antibodies were incubated at RT for $1 \mathrm{~h}$, washed in TBST and developed using ECL systems (GE Healthcare). The primary antibodies used are polyclonal goat anti-TrkB (AF1494, Bio-Techne Sales Corp; 1:500) and monoclonal mouse anti-atubulin (T5168, Sigma-Aldrich; 1:5000). The secondary antibodies used are peroxidase AffiniPure donkey 
anti-rabbit IgG $(\mathrm{H}+\mathrm{L})$ (711-035-152, Jackson ImmunoResearch; 1:10,000), peroxidase AffiniPure donkey anti-goat IgG $(\mathrm{H}+\mathrm{L})$ (705-035-003, Jackson ImmunoResearch; 1:10,000) and peroxidase AffiniPure goat anti-mouse IgG $(H+L)$ (115-035-146, Jackson ImmunoResearch; 1:10,000).

\section{Quantitative qRT-PCR}

Total RNA was extracted separately from somatodendritc and axonal compartments and reversetranscribed using random hexamers and Superscript III Reverse transcriptase enzyme (18080044; Invitrogen), as described previously [20]. cDNA was purified using QIAGEN II purification kit (20021). For qRT-PCR, Luminaris HiGreen qPCR Master Mix (Thermo Fisher Scientific) was applied on a lightCycler ${ }^{\circledR}$ 96 thermal cycler (Roche). Histone $\mathrm{H} 1 \mathrm{fO}$ transcripts were absent in RNA fractions obtained from axonal compartments, confirming the purity of mRNA preparation from these compartments. GAPDH was used for data normalization. The following primers were used for qRT-PCR: TrkB: 5'-

CGGGAGCATCTCTCGGTCTAT-3' (forward) and 5'-CTGGCAGAGTCATCGTCGTTG-3' (reverse); Gapdh: 5'AACTCCCACTCTTCCACCTTC-3' (forward), and 5'-GGTCCAGGGTTTCTTACTCCTT-3' (reverse); and histone H1f0, 5'-CCCAAGTATTCAGACATGAT-3' (forward), and 5'-CGCTTGATGGACAACT-3' (reverse).

\section{Results}

\section{ER dynamics are regulated by a coordinated actin/microtubule cytoskeleton in growth cones of cultured motoneurons}

In growth cones of rat sympathetic neurons, membrane-bound organelles representing ER often colocalize with microtubules and hardly extend beyond microtubules [22]. Nevertheless, growth cone filopodia are enriched in actin filaments and mostly lack microtubules [23]. Thus, we wondered whether presynaptic ER could extend into these actin-rich filopodia and associate with actin filaments in addition to microtubules. In order to visualize the presynaptic ER, we transduced primary cultured motoneurons with a lentivirus expressing mCherry-KDEL, a well-studied ER marker [24]. Neurons were then fixed and immunostained against F-actin and microtubule cytoskeleton using phalloidin and a-tubulin staining, respectively. The association of presynaptic ER with actin and tubulin was then assessed using Structured Illumination Microscopy (SIM) (Fig. 1a). Interestingly, the presynaptic ER was detected not only in the core of growth cones but also in filopodia (Fig. 1b). Notably, ER colocalized with both F-actin and microtubules in the core, while in filopodia, ER overlapped mostly with F-actin, as expected since microtubules are less abundant in filopodia (Fig. 1b, c). To confirm the colocalization of the presynaptic ER with F-actin in filopodia, we monitored the dynamics of ER/actin co-movements in filopodia by transducing motoneurons with a GFP-actin-IRES-mCherry-KDEL lentivirus that allows co-expression of GFP-actin and mCherry-ER. We captured live cell imaging of growth cones from motoneurons that were transduced with GFP-actin-IRES-mCherry-KDEL over 8 min with $2 \mathrm{sec}$ intervals to visualize actin and ER movements simultaneously (Fig. 1d). Two color live cell imaging demonstrated that tubular ER extends into filopodia and retracts along actin filaments indicating that ER interaction with actin defines its rearrangement and remodeling in filopodia (Fig. 1d, Video 1). Representative multiple kymographs 
indicated coordinated movements of actin and ER in filopodia (Fig. 1e). Based on these observations, we hypothesized that ER extension into filopodia might be actin and not microtubule dependent. To address this, we transduced motoneurons with mCherry-KDEL and treated them with actin and microtubule depolymerizing drugs cytochalasin $\mathrm{D}(\mathrm{CytoD})$ or nocodazole, respectively. ER movements were then evaluated in the growth cone by live cell imaging (Fig. 2a, b, Video 2-7). To measure ER dynamic movements, we implemented an adaption of Image Correlation Spectroscopy (ICS) [25] (Fig. 2C). In addition to ICS-analysis, we used multiple kymographs to measure the frequency and distance moved by presynaptic ER manually. Intriguingly, we found that the dynamic movement of presynaptic ER in filopodia is significantly higher than that in the core (Fig. 2d). Besides, the velocity of dynamic movements was higher, as represented in Fig. 2e, and the distance moved by ER in filopodia was greater compared to growth cone core, correspondingly (Fig. 2f). Moreover, we found that upon CytoD treatment, $80 \%$ of neurons failed to show ER movements in filopodia, while only $40 \%$ failed to show ER movements in the core (Fig. $2 \mathrm{~g}$ ). Treatment with nocodazole resulted in 50\% failure in filopodia and $40 \%$ failure in core ER movements (Fig. 2g). ICS-analysis revealed that disruption of either actin or microtubule dynamics reduces ER movements significantly in both filopodia and core implying that ER dynamic movements depend on a coordinated actin/microtubule cytoskeleton (Fig. 2h, i). Nevertheless, analyzing the frequency of ER movements in filopodia demonstrated that disruption of actin but not microtubules reduced the frequency of ER movements (Fig. 2h). In contrast to filopodia, the frequency of ER movements in core was markedly reduced upon disruption of either actin or microtubule cytoskeleton which is in agreement with the above ICS-analysis (Fig. 2i). This finding was further confirmed by a treatment with both CytoD and nocodazole which severely impaired the ER dynamics in both filopodia and core, as nearly no more ER movements were detectable (Fig. 2g, Supplementary Figure 1A).

Collectively, these data provide evidence of a highly dynamic ER in distal axons that also includes presynaptic compartments of developing motoneurons. The dynamic movements of ER in axonal growth cones could be classified into fast movements in filopodia and slower movements in the core. Fast ER remodeling in filopodia is regulated particularly by actin cytoskeleton, whereas slow ER rearrangements in the core require a microtubule and actin crosstalk.

\section{Extracellular stimulation triggers ribosome activation and initiates local translation in growth cones on a time scale of seconds}

Deep RNA-seq combined with sensitive fluorescence in situ hybridization (FISH) approaches have identified numerous mRNAs $[26,27]$ within distal axons which are locally translated $[20,28,29]$. Locally synthetized proteins are necessary for neural-circuit development, survival and plasticity. In developing axons, local translation mediates an essential response to guidance cues required for pathfinding [30]. Brain-derived neurotrophic factor (BDNF) and its receptor TrkB play a vital role in modulation of local translation [31] and axonal cytoskeleton remodeling [32,33] in motoneurons. We sought to scrutinize the dynamics of ribosome activation in response to BDNF stimulation in the growth cone of motoneurons. Following BDNF binding, TrkB undergoes autophosphorylation and activates MAPK as well as PI3K-AKT signaling pathways [34], leading to activation of ribosomes and induction of local translation [35]. In 
order to define the precise kinetics of TrkB activation, we applied BDNF to motoneurons with a short pulse of $10 \mathrm{sec}, 1 \mathrm{~min}$ and $10 \mathrm{~min}$, washed it out and immunostained neurons against TrkB and pTrkB. The specificity of TrkB and pTrkB antibodies were confirmed by immunostaining using TrkB knockout mice (Supplementary Figure 2A, B). We could detect phosphorylated TrkB upon $10 \mathrm{sec}$ BDNF pulse in the growth cone, as shown by immunofluorescence assay (Fig. 3a, b). Interestingly, the levels of TrkB also elevated in the growth cone after 1 min BDNF exposure (Fig. $3 \mathrm{C}$ ). In the whole cell lysate, a corresponding elevation of pTrkB immunoreactivity became detectable first at 1 min poststimulation, as shown by Western blot (Fig. 3d) and no increase in total levels of TrkB was detectable despite 10 min stimulation, indicating that this short pulse is insufficient to induce the transcription and translation of TrkB in the soma (Fig. 3d, Supplementary Figure 2C). The rapid increase in TrkB immunoreactivity within less than 1 min in the growth cone of immunostained motoneurons could be explained by release of this receptor from intracellular stores or changes in the receptor conformation, which favors antibody binding. The increase in TrkB immunoreactivity in growth cones at a later time point of 10 min poststimulation (Fig. 3c) could be explained by enhanced transport of TrkB from distal axons into growth cones and/or rapid neosynthesis in growth cones since TrkB mRNAs were detected in axons by qRT-PCR assay (Supplementary Figure 2D). Treatment of neurons with either anisomycin, which inhibits the translation, or nocodazole, which blocks the microtubule-based transport, prevents the increase in TrkB signal in growth cones (Fig. $3 e, f)$. These data indicate that BDNF stimulation triggers redistribution of TrkB resulting in an increase in TrkB total immunofluorescence within $1 \mathrm{~min}$, and also induces its local production within 10 min stimulation.

In developing axons, the rapid response to extracellular cues is assured by tight regulation of spatiotemporal changes in mRNA translation $[36,37]$. Thus, the kinetics of induction of ribosomal changes that trigger translation in growth cones should differ from those in the soma. To study the dynamics of ribosome remodeling, we used Y10B as a general marker for ribosomes and RPL8 as a marker for the $60 \mathrm{~S}$ ribosomal subunit in order to study the dynamics of movement of these different subunits. We found that induction of BDNF/TrkB signaling leads to a rapid change in the distribution of these ribosomal markers within the growth cone. The immunoreactivities of ribosomal markers $\mathrm{Y} 10 \mathrm{~B}$ and RPL8 were rapidly altered and appeared increased after $10 \mathrm{sec}$ BDNF pulse (Fig. 3g-i). Interestingly, short pulses of $10 \mathrm{sec}$ and $1 \mathrm{~min}$ were not sufficient to induce such changes in ribosome distribution in the soma as shown in Supplementary Figure 2E, albeit TrkB phosphorylation was detectable also in this subcellular compartment after $10 \mathrm{sec}$ stimulation (Supplementary Figure 2F). This kinetic distinction suggests that in the growth cone, BDNF/TrkB signaling and downstream mechanisms for modulating ribosome distribution and possibly also activation are different from cell bodies. Increased immunoreactivity for ribosomal subunits in the growth cone within $10 \mathrm{sec}$ BDNF stimulation is striking. Rapid transport of ribosomal subunits from axonal sites to the growth cone appears unlikely since the fastest measured microtubule-dependent axonal transport at a speed of $1 \mu \mathrm{m} / \mathrm{sec}$ cannot provide ribosomes from the axon shaft into growth cones in such a short time [38]. To exclude this, we treated neurons with nocodazole to disrupt microtubule-based axonal transport and found that disruption of axonal transport did not affect BDNF-induced augmentation of ribosomal subunits as illustrated in Fig. 
3j. Similarly, inhibition of translation by anisomycin treatment results in a significant increase in ribosome immunoreactivity, indicating that this increase does not depend on de novo synthesis of ribosomal proteins within the growth cone (Fig. 3k). Strikingly, CytoD treatment completely prevents the ribosomal response to BDNF stimulation, as no increase in the signal intensities of Y10B and RPL8 were detectable upon either stimulation times (Fig. $3 \mathrm{l}, \mathrm{m}$ ). Based on these observations, we propose that elevated immunoreactivity of ribosomes within $10 \mathrm{sec}$ stimulation might be due to actin dependent conformational and distribution changes in ribosomal subunits which occurs upon assembly of $80 \mathrm{~S}$ subunits and thus alters accessibility of corresponding epitopes for detection with Y10B and RPL8 antibodies. To investigate this hypothesis, we stained motoneurons with antibodies against RPL24 of the 60S and RPS6 of the 40S subunits (Fig. 4a). Upon assembly of $80 \mathrm{~S}$ ribosomes, the C-terminus of RPL24 interacts with RPS6, thereby forming a bridge between the two subunits [39]. We employed SIM with a resolution of $\sim 120 \mathrm{~nm}$ to examine the interaction of RPL24/RPS6 in growth cones. These experiments were performed both with CD1 WT motoneurons (Supplementary Figure 1B) and Smn+/+; SMN2tgtg motoneurons for a better comparability with Smn-deficient motoneurons used for the experiment shown in Fig. 9. As depicted in Fig. 4b, within $10 \mathrm{sec}$ of stimulation the co-clusters of RPL24/RPS6 increased by 3 -fold compared to no stimulation condition. Thus, we reasoned that the rapid increase in $\mathrm{Y} 10 \mathrm{~B}$ immunoreactivity in response to extracellular stimulation involves extremely fast assembly of ribosomes into $80 \mathrm{~S}$ subunits. The increased interaction of RPL24 and RPS6 ribosomal proteins and consequently the assembly of $80 \mathrm{~S}$ ribosomes suggest that this conformational change could correlate with translation initiation. To visualize actively translating ribosomes, we co-stained neurons against $\mathrm{Y} 10 \mathrm{~B}$ as a structural component of ribosomes and the elongation factor eEF2, which associates only with actively translating ribosomes (Fig. 4c). Again, we found a significant increase in the number of Y10B/eEF2 co-clusters within $10 \mathrm{sec}$ of stimulation, indicating that the response of ribosomes in growth cones to activation of TrkB receptors is very fast (Fig. 4d). For a direct comparison of Smnt/t;SMN2tgtg motoneurons with motoneurons from CD1 mice that were used as WT controls in other experiments, we counted the coclusters of RPL24/RPS6 as well as Y10B/eEF2 in motoneurons obtained from mice with CD1 genetic background. We observed a significant increase in RPL24/RPS6 co-clusters at 10 sec poststimulation and a tendency at 1 min poststimulation (Supplementary Figure 1B). Likewise, co-clusters of Y10B/eEF2 were increased significantly within $10 \mathrm{sec}$ as well as $1 \mathrm{~min}$ BDNF stimulation (Supplementary Figure 1C). To confirm that the rapid activation of ribosomes depends on BDNF/TrkB signaling, we stained motoneurons from TrkB knockout mice against $\mathrm{Y} 10 \mathrm{~B}$ and found that in TrkB knockout motoneurons ribosomes fail to respond to BDNF stimulation (Supplementary Figure 2G, H). Next, we questioned whether this rapid activation of ribosomes in response to extracellular stimuli associates with a temporal change in protein synthesis in axonal growth cones. To examine the dynamics of local translation, we applied a time series after BDNF pulse to motoneurons, fixed them and stained against b-actin (Fig. 5a). Remarkably, we observed a significant increase in b-actin signal intensity within 1 min of stimulation suggesting that either the velocity of protein synthesis is extremely high in growth cones or actin undergoes conformational changes in such a way that this leads to enhanced immunoreactivity (Fig. 5b). To distinguish these two possibilities and to confirm that elevated levels of b-actin rely on protein synthesis and not transport from the axon, we pretreated neurons with anisomycin and nocodazole and 
applied BDNF pulses for $10 \mathrm{sec}$ to $10 \mathrm{~min}$. Inhibition of protein synthesis prevented the increase of b-actin protein levels, while disruption of microtubule-dependent axonal transport had no influence (Fig. $5 c$, d). In addition, we used puromycin to measure the rate of newly synthetized proteins (Fig. 5e, f). Similar to bactin, within 1 min of stimulation, synthesis of de novo proteins was accomplished as detected by marked increase in puromycin immunoreactivity, supporting the observation of an extremely fast rate of local translation in growth cones (Fig. $5 \mathrm{~g}$ ). In contrast, 1 min likewise 10 min stimulations did not trigger translation in the soma under the same conditions, pointing again towards different translation kinetics in these distinct compartments (Supplementary Figure 2I). In line with that, inhibiting translation but not axonal transport prohibited elevated protein synthesis in response to extracellular stimuli as demonstrated by the puromycin assay (Fig. 5h, i). Taken together, these data indicate that in presynaptic compartments, BDNF/TrkB signaling activates the translational machinery within seconds and thus regulates local protein synthesis at an extraordinary high speed.

\section{Rough ER is present in the presynaptic compartment and contributes to Brain-derived neurotrophic factor induced presynaptic protein translation}

Transcripts encoding membrane and secreted proteins as well as ER resident proteins have been reported to be present in axons, and the corresponding proteins are synthetized and delivered into the axoplasmic membrane [36, 40,41]. Although initial ultrastructural approaches conducted more than 40 years ago have only identified smooth ER in neuronal processes [17], fluorescence microscopy approaches have identified ER-associated proteins implicated in protein translocation, folding, and post-translational modifications as well as proteins of the Golgi apparatus in distal axons suggesting that axons might contain RER [18]. Accordingly, we considered whether the presynaptic compartment harbors RER, thereby participating in processing of locally produced membrane and secretory proteins. To address this hypothesis, we transduced Smn+/+; SMN2tgtg motoneurons with lentiviruses encoding the ER marker mCherry-KDEL and co-stained against RPL24 and RPS6 as markers of entirely assembled 80S ribosomes (Fig. 6a). In contrast to unstimulated neurons, a significant number of RPL24/RPS6 co-clusters colocalized with ER in presynaptic compartments of stimulated neurons, and this colocalization increased after $10 \mathrm{sec}$ as well as 1 min BDNF pulses (Fig. 6b). Next, we examined the attachment of actively translating ribosomes on presynaptic ER by staining mCherry-KDEL expressing neurons against Y10B and eEF2 as depicted in Fig. 6c. Similarly, $10 \mathrm{sec}$ and 1 min stimulations caused an increase in colocalization of $\mathrm{Y} 10 \mathrm{~B} / \mathrm{eEF} 2$ co-clusters with presynaptic ER indicating that ribosomes in the elongation stage of translation attach to the presynaptic ER (Fig. 6d). To confirm these results in vivo, we stimulated NMJs of P5 WT mice with BDNF and performed IHC using an ER marker, calreticulin, and Y10B as ribosomal marker (Fig. 6e). Consistent with in vitro data, we could detect ribosomes which associated with ER within the presynaptic area labeled by synaptophysin. These findings imply that RER exists both in the growth cone of developing neurons and in the nerve terminals in vivo and thus support a role for presynaptic ER in processing and surface delivery of axonally synthetized membrane and secretory proteins. 


\section{Altered regulation of dynamics of local translation and aberrant presynaptic ER dynamics are involved in axon pathology in spinal muscular atrophy}

As demonstrated by previous studies [21, 42, 43], aberrant axonal mRNA localization and impaired local translation are important contributors to disease manifestation and clinical pathology in SMA and ALS. Therefore, we sought to investigate the importance of our observations on dynamic regulation of local translation and contribution of the presynaptic ER in context of SMA. We used a mouse model that resembles severe type I SMA in humans. First, we measured the time course of BDNF-induced ribosome activation using Y10B immunostaining in Smn-deficient motoneurons (Fig. 7a). Interestingly, even 10 min stimulation did not elicit any alteration in ribosome distribution in Smn-deficient growth cones as the fluorescence intensity for Y10B did not increase, in contrast to WT control motoneurons (Fig. 7b). In line with that, we did not detect increased immunoreactivity of TrkB as a response to BDNF stimulation, indicating that the mechanisms that underly these changes in wildtype cells are defective in Smndeficient motoneurons (Fig. 7c, d). Total levels of TrkB were similar in control and Smn-deficient neurons under basal (no BDNF stimulation) condition in both growth cones and soma (Fig. 7e). BDNF-stimulated Smn-deficient motoneurons also failed to show alterations in puromycin immunofluorescence indicating that lack of Smn protein could be responsible for disturbed kinetics of local translation and impaired dynamic regulation of growth cone proteome (Fig. 7c, f). Next, we measured dynamic movements of presynaptic ER in Smn-deficient motoneurons and found that while dynamic movements are not disturbed in the growth cone core, the movements are markedly reduced in filopodia (Fig. 8a, b, Video 89). Consistent with these defects, SIM image analyses of ER colocalization with F-actin and a-Tubulin in growth cones of Smn-deficient motoneurons revealed that the altered distribution of actin filaments in filopodia correlates with disturbed ER entry into filopodia, which could explain the mechanism underlying defective ER dynamic remodeling in growth cone filopodia (Fig. 8c).

Finally, we investigated the dynamics of ribosome activation and their association to presynaptic ER upon BDNF induction by SIM. We found that in Smn-deficient motoneurons, BDNF stimulation had no or only minor effects below detection limit on the assembly of $80 \mathrm{~S}$ ribosomes (Fig. 9a). As shown in Fig. 9b, we did not detect elevated levels of RPL24/RPS6 co-clusters in BDNF-stimulated Smn-deficient motoneurons compared to unstimulated Smn-deficient neurons. Smn+/t; SMN2tgtg motoneurons showed elevated levels of RPL24/RPS6 co-clusters upon BDNF induction compared to unstimulated Smn+/+; SMN2tgtg neurons (Fig. 9b and Fig. 4b). Likewise, the number of RPL24/RPS6 co-clusters associated with ER was not altered in growth cones of BDNF-stimulated Smn-deficient motoneurons compared to unstimulated Smn-deficient neurons (Fig. 9a, c). To confirm these results, we performed immunostaining with $\mathrm{Y} 10 \mathrm{~B}$ and eEF2 in mCherry-ER expressing motoneurons to evaluate ER and ribosomes and their association in Smn-deficient motoneurons (Fig. 9d). Compared to unstimulated Smndeficient neurons, BDNF-stimulated Smn-deficient neurons did not show increased levels of Y10B/eEF2 co-clusters (Fig. 9e). In contrast, BDNF-stimulated Smn+/t; SMN2tgtg motoneurons displayed significant increase in RPL24/RPS6 co-clusters that associated with ER compared to unstimulated Smn+/+; SMN2tgtg neurons (Fig. 9b), as previously illustrated in Fig. 4b. Furthermore, no enhanced levels of Y10B/eEF2 co-clusters associated with ER that are prominent in Smn+/t; SMN2tgtg neurons (Fig. 6d, Fig. 
9f) were detected in Smn-deficient neurons after a $10 \mathrm{sec}$ or 1 min BDNF pulse (Fig. 9f). Thus, Smn deficiency leads to impaired BDNF/TrkB responses both in ER dynamics and in ribosome assembly, which in turn could cause disturbed translation initiation and ER attachment of ribosomes in the presynaptic compartment.

\section{Discussion}

In neurons, ER forms a highly dynamic network in dendrites and axons. The regulation of axonal ER dynamic movements as well as its function in development and maintenance of synapses have gained emerging interest [44-46]. In this study, we developed a technique to visualize and quantify the dynamic movements of ER in presynaptic terminals of cultured motoneurons and show that motoneurons harbor a rough ER in the axonal growth cone which extends into filopodia. Its integrity and dynamic remodeling are regulated mainly by the actin cytoskeleton, and this dynamic remodeling is markedly disturbed in motoneurons from a mouse model of spinal muscular atrophy.

As apposite to microtubules [24], the interaction of ER with actin cytoskeleton especially in the axon and presynaptic area has not been investigated yet in much detail. In somatodendritic compartments, kinesin and dynein-based ER sliding along microtubules as well as interaction between STIM1 on the ER and EB1 on the microtubule plus end have shown to mediate ER translocation $[3,47]$. However, studies with cultured cells have revealed that first, nocodazole treatment does not retract ER from periphery immediately [48], second, ER tubules can form in the absence of microtubules [49-51] and third, disruption of actin filaments in hippocampal neurons affects $\mathrm{Ca}^{2+}$ release from ER in soma [52]. These observations indicate that ER dynamic movements might not depend only on microtubules but also on actin cytoskeleton. Indeed, the first indirect evidence for the role of actin in ER translocation into dendrites comes from a study by Wagner et al., showing that myosin-Va, which is an actin-based motor, mediates ER movement in dendritic spines of Purkinje neurons [7]. Axonal growth cones consist of highly dynamic membrane protrusions; "filopodia and lamellipodia", and a more stable central region; "growth cone core" [53]. All these domains are transient and undergo constant growth and retraction in shape and structure [54]. In contrast to microtubule-rich core, actin is predominant in filopodia and lamellipodia. Therefore, dynamics of filopodia exceed those in the core [55]. In line with that, our time-lapse recordings show that the dynamics of ER differs in growth cone center and periphery. In the core of growth cones, the velocity of ER dynamic movements is much lower than that in actin-rich filopodia. Importantly, we found that ER movements in the core are regulated by both actin and microtubule cytoskeleton. Previous studies have demonstrated that F-actin and microtubules interplay during neuronal polarization and development [5658]. This crosstalk is mediated by macromolecules such as Drebrin E, which physically links F-actin and microtubule plus end binding protein or other regulatory proteins such as Rho GTPases, MAP2 and tau $[56,58]$. Thus, such mechanisms could be implicated in the coordinated actin/microtubule regulation of ER dynamics in the growth cone core. Strikingly, actin depolymerization destroys ER dynamic movements in filopodia of $80 \%$ of imaged cells. In addition, two-color live cell imaging data revealed that ER moves along actin in filopodia and F-actin withdrawal results in nearly $99 \%$ retraction of ER from filopodia. 
These data provide the first direct evidence of an actin-dependent ER remodeling and integrity in axon terminals of neurons. ER entry into filopodia suggests a membrane contact site between the ER and the plasma membrane [1]. This physical tethering could be essential for the surface delivery of newly synthesized receptors, membrane proteins or lipids. Further studies are required to investigate whether myosin-Va might be also involved in such actin-dependent ER dynamic regulation in axons. Regulation of dynamic remodeling of axonal ER recently became attentive through studies identifying mutations in ER regulatory proteins that associate with human neurodegenerative diseases including hereditary spastic paraplegia, Alzheimer's disease (AD) and ALS. Mutations in ER-shaping proteins such as Spastin, ATL1, RTN2 or REEP1 that couple tubular ER network with microtubule dynamics cause hereditary spastic paraplegia [59,60]. AD patients carry mutations in Rab10, another protein involved in microtubule and ER coupling [61, 62]. Similarly, mutations in vesicle-trafficking protein A and B (VAPA and VAPB) cause lateonset SMA and ALS $[8,63]$. VAPA and VAPB proteins reside in ER and facilitate anchoring of membranous organelles to the ER [8] and regulate tubular ER morphogenesis and dynamics [46]. Such mutations that impair ER dynamic remodeling do not only disturb ER functions but also impact mitochondria and endosomes through ER MCSs [10]. Our data unravel that loss of SMN protein associates with impaired dynamic remodeling of ER in actin-rich growth cone filopodia in cultured motoneurons. Interestingly, we did not observe defective ER dynamics in the growth cone core of Smndeficient motoneurons, indicating that impaired actin and not microtubule cytoskeleton underlies the defective ER dynamics in the growth cone of Smn-deficient motoneurons. In line with that, SIM data show altered colocalization of ER with F-actin in growth cone filopodia of Smn-deficient motoneurons, again confirming disturbed actin-dependent ER extension into filopodia. Actin cytoskeleton appears to play a central role in neurodegeneration through involvement in key axonal functions and synapse maintenance [64]. Indeed, there is emerging evidence for a defective actin cytoskeleton in axons and presynaptic terminals of SMA patients as well as animal models. Loss of SMN causes impaired mRNA localization and local translation of actin isoforms Acta and Act $\beta$ resulting in disturbed dynamic of F-actin polymerization in the growth cone [20]. Profilin-2 is a neuronal specific regulator of F-actin polymerization which has been shown to directly interact with SMN protein $[65,66]$. SMN interaction with Profilin-2 reduces its sequestering activity on F-actin, thereby promoting actin polymerization, whereas SMN release allows Profilin-2 binding to actin monomers, leading to reduced actin polymerization [67]. Plastin-3, an actin bundling protein that also regulates actin turnover, is a protective modifier of SMA and its overexpression has been shown to ameliorate defects in axon growth [68], NMJ maturation and motor functions in SMA animal models [69]. Notably, SMA mouse models exhibit elevated levels of microtubules and neurofilaments in the presynaptic terminals [70]. Therefore, a compensatory mechanism involving microtubules could be considered for the observed intact dynamic remodeling of ER in the growth cone core of Smn-deficient motoneurons.

Subcellular control of axon growth, synapse differentiation and plasticity depend on regulatory mechanisms that ensure processing of local information and subsequent responses on a time scale of seconds. mRNA localization and local protein synthesis are conserved mechanisms that modify axonal proteome at a fast temporal and spatial scale, thereby maintaining plasticity capacity of axonal 
synapses [27]. In addition to cytoplasmic proteins, mRNAs encoding secreted and membrane proteins have been identified in axonal transcriptomes $[21,36,71]$. On-site synthesis and glycosylation of such membrane proteins would require additional RER and Golgi machinery in the distal axon. However, previous studies have suggested that in axons ER is considered to be devoid of ribosomes and thus no direct evidence of its potential functions related to local protein synthesis has been reported yet $[11,16$, 17]. Interestingly, Merianda et al., 2009 reported glycosylation patterns of transmembrane proteins being locally translated in axons of sensory neurons [18]. We used a BDNF pulse approach combined with super-resolution fluorescence imaging by SIM and demonstrated that stimulation of motoneurons triggers the assembly of $80 \mathrm{~S}$ ribosomes and initiates the translation in axon terminals within $10 \mathrm{sec}$. Ribosomes in the elongation phase of translation attach to the presynaptic ER and locally synthetized proteins become detectable at 1 min poststimulation. Contrary to axons, the response of soma to BDNF/TrkB activation was considerably slower and happened in longer time scales of several minutes. These distinctive response rates could be due to different intracellular levels of second messengers such as CAMP [72]. It remains unknown whether similar or distinct signaling pathways downstream to BDNF/TrkB, such as mTOR, MAPK and PI3K pathways, are involved in activation of ribosomes and translation initiation in the growth cone and its counterparts. Thus, our data uncover the rate of dynamic local translation at presynaptic terminals of cultured motoneurons and identify a rough ER in axon terminals that participates in post-translational processing of locally synthetized proteins in response to extracellular stimuli. This fast rate of translation indicates that ribosomes only transiently associate with presynaptic ER in response to stimuli and explains why previous studies failed to detect RER in axons using unstimulated tissues.

Translational defects and disturbed mRNA recruitment onto polysomes previously reported in SMA mouse models implicate a master function of SMN in translational regulation [20,73, 74]. Indeed, SMN is not only involved in the assembly of snRNP particles but also associates directly with actively translating polysomes, as shown in cultured cells, spinal cord and brain tissues $[75,76]$. In our study, Smn-deficient motoneurons failed to respond to BDNF stimuli by eliciting the fast assembly of $80 \mathrm{~S}$ ribosomes, resulted in delayed translation initiation and perturbed association of ribosomes with the presynaptic ER after BDNF stimulation. In motoneurons, BDNF modulates diverse neuronal functions such as survival, growth and differentiation [77-79]. Strikingly, we did not detect any reduction in TrkB levels in both growth cone and soma of Smn-deficient motoneurons. In WT motoneurons, TrkB levels elevate in the growth cone within 1 min after BDNF stimulation, which is indicative of a rapid increase of cell surface translocation of TrkB receptors. This so called "self-enhancement" of BDNF-TrkB signaling seems to be critical for synaptic plasticity and maintenance [80]. Notably, upon BDNF pulse the levels of detectable TrkB immunoreactivity did not increase in Smn-deficient motoneurons even at 10 min poststimulation. Interestingly, CytoD treatment has been shown to inhibit TrkB translocation to the cell surface [81]. Our data also show that in growth cones of WT neurons, CytoD-treatment abolishes the ribosomal response to BDNF pulse, indicating that an actin-based motor moves ribosomes and possibly tethers them to the ER. Thus, an impaired actin cytoskeleton affecting cell surface TrkB translocation, ER dynamic 
remodeling and ribosome/ER anchoring appears to underlie the mechanism of disturbed BDNF responsivity and synaptic defects in SMA.

\section{Conclusions}

In summary, this work identifies a novel function for axonal ER in regulation of stimulus-induced local translation and discloses the mechanism of dynamic regulation of presynaptic ER by an actinmicrotubule crosstalk. Our study on Smn-deficient motoneurons uncovers molecular determinants involved in impaired sensing of changes in the extracellular environment leading to a delayed response and explains why SMN deficiency makes motoneurons vulnerable to axon and synapse differentiation aberrations.

\section{Declarations}

\section{Ethics approval and consent to participate}

Not applicable.

\section{Consent for publication}

All authors read and approved the final manuscript.

\section{Availability of data and materials}

The primary datasets used and/or analyzed during the current study are available from the corresponding author on reasonable request.

\section{Competing interests}

The authors declare no competing financial interests.

\section{Funding}

Chunchu Deng was supported by a grant provided from PicoQuant. PicoQuant was not involved in design, performing or analysis of data. This paper received further support from the DFG, Grant Se697/71, project number 405988308.

\section{Authors' contributions}

CCD, MM and M. Sendtner conceived the project, designed the experiments and wrote the manuscript. CCD performed all the experiments including live cell imaging of ER dynamics, motoneuron cultures, ICC, IHC, SIM and confocal microscopy and data analysis. MM performed qRT-PCR and compartmentalized motoneuron cultures. SR and SD performed analysis of ER dynamic movements. MM, LH and SJ helped with confocal image acquisition and data analysis. M. Sauer gave support with super resolution 
microscopy. LH provided additional data for discussions. All authors read and approved the final manuscript.

\section{Acknowledgments}

We thank Markus Behringer for assistance with SIM, Regine Sendtner for the organization of animal breeding, Hildegard Troll for lentivirus production and Dr. Robert Blum and Dr. Patrick Lüningschrör for helpful discussions.

\section{Authors' information}

${ }^{*} \mathrm{CC}$. Deng and M. Moradi contributed equally to this work.

Corresponding author

Correspondence to Michael Sendtner at Sendtner_M@ukw.de.

\section{Abbreviations}

AD: Alzheimer's Disease

ALS: Amyotrophic Lateral Sclerosis

BDNF: Brain-derived Neurotrophic Factor

ER: Endoplasmic Reticulum

HSP: Hereditary Spastic Paraplegia

NMJs: Neuromuscular Junctions

SIM: Structured Illumination Microscopy

SMA: Spinal Muscular Atrophy

SMN: Survival of Motor Neuron

TVA: Transverse Abdominal muscle

TrkB: Tropomyosin receptor kinase B

\section{References}

1. Wu, Y., et al., Contacts between the endoplasmic reticulum and other membranes in neurons. Proc Natl Acad Sci U S A, 2017. 114(24): p. E4859-E4867. 
2. Terasaki, M., et al., Continuous network of endoplasmic reticulum in cerebellar Purkinje neurons. Proc Natl Acad Sci U S A, 1994. 91(16): p. 7510-4.

3. Waterman-Storer, C.M. and E.D. Salmon, Endoplasmic reticulum membrane tubules are distributed by microtubules in living cells using three distinct mechanisms. Curr Biol, 1998. 8(14): p. 798-806.

4. Griffing, L.R., Networking in the endoplasmic reticulum. Biochem Soc Trans, 2010. 38(3): p. 747-53.

5. Prinz, W.A., et al., Mutants affecting the structure of the cortical endoplasmic reticulum in Saccharomyces cerevisiae. J Cell Biol, 2000. 150(3): p. 461-74.

6. Farias, G.G., et al., Feedback-Driven Mechanisms between Microtubules and the Endoplasmic Reticulum Instruct Neuronal Polarity. Neuron, 2019. 102(1): p. 184-201 e8.

7. Wagner, W., S.D. Brenowitz, and J.A. Hammer, 3rd, Myosin-Va transports the endoplasmic reticulum into the dendritic spines of Purkinje neurons. Nat Cell Biol, 2011. 13(1): p. 40-8.

8. Teuling, E., et al., Motor neuron disease-associated mutant vesicle-associated membrane proteinassociated protein (VAP) B recruits wild-type VAPs into endoplasmic reticulum-derived tubular aggregates. J Neurosci, 2007. 27(36): p. 9801-15.

9. Blackstone, C., Cellular pathways of hereditary spastic paraplegia. Annu Rev Neurosci, 2012. 35: p. 25-47.

10. Ozturk, Z., C.J. O'Kane, and J.J. Perez-Moreno, Axonal Endoplasmic Reticulum Dynamics and Its Roles in Neurodegeneration. Front Neurosci, 2020. 14: p. 48.

11. Horton, A.C. and M.D. Ehlers, Dual modes of endoplasmic reticulum-to-Golgi transport in dendrites revealed by live-cell imaging. J Neurosci, 2003. 23(15): p. 6188-99.

12. Lee, J.E., et al., Endoplasmic reticulum contact sites regulate the dynamics of membraneless organelles. Science, 2020. 367(6477).

13. Puhka, M., et al., Progressive sheet-to-tubule transformation is a general mechanism for endoplasmic reticulum partitioning in dividing mammalian cells. Molecular biology of the cell, 2012. 23(13): $p$. 2424-2432.

14. Shibata, Y., et al., Mechanisms determining the morphology of the peripheral ER. Cell, 2010. 143(5): p. 774-88.

15. West, M., et al., A 3D analysis of yeast ER structure reveals how ER domains are organized by membrane curvature. Journal of Cell Biology, 2011. 193(2): p. 333-346.

16. Krijnse-Locker, J., et al., The organization of the endoplasmic reticulum and the intermediate compartment in cultured rat hippocampal neurons. Mol Biol Cell, 1995. 6(10): p. 1315-32.

17. Tsukita, S. and H. Ishikawa, Three-dimensional distribution of smooth endoplasmic reticulum in myelinated axons. J Electron Microsc (Tokyo), 1976. 25(3): p. 141-9.

18. Merianda, T.T., et al., A functional equivalent of endoplasmic reticulum and Golgi in axons for secretion of locally synthesized proteins. Mol Cell Neurosci, 2009. 40(2): p. 128-42.

19. Jablonka, S. and M. Sendtner, Molecular and cellular basis of spinal muscular atrophy. Amyotroph Lateral Scler Other Motor Neuron Disord, 2003. 4(3): p. 144-9. 
20. Moradi, M., et al., Differential roles of alpha-, beta-, and gamma-actin in axon growth and collateral branch formation in motoneurons. J Cell Biol, 2017. 216(3): p. 793-814.

21. Saal, L., et al., Subcellular transcriptome alterations in a cell culture model of spinal muscular atrophy point to widespread defects in axonal growth and presynaptic differentiation. RNA, 2014. 20(11): p. 1789-802.

22. Dailey, M. and P. Bridgman, Dynamics of the endoplasmic reticulum and other membranous organelles in growth cones of cultured neurons. The Journal of Neuroscience, 1989. 9(6): p. 18971909.

23. Geraldo, S. and P.R. Gordon-Weeks, Cytoskeletal dynamics in growth-cone steering. J Cell Sci, 2009. 122(Pt 20): p. 3595-604.

24. Guo, Y., et al., Visualizing Intracellular Organelle and Cytoskeletal Interactions at Nanoscale Resolution on Millisecond Timescales. Cell, 2018. 175(5): p. 1430-1442.e17.

25. Wiseman, P.W., Image correlation spectroscopy: principles and applications. Cold Spring Harb Protoc, 2015. 2015(4): p. 336-48.

26. Briese, M., et al., Whole transcriptome profiling reveals the RNA content of motor axons. Nucleic Acids Res, 2016. 44(4): p. e33.

27. Holt, C.E., K.C. Martin, and E.M. Schuman, Local translation in neurons: visualization and function. Nat Struct Mol Biol, 2019. 26(7): p. 557-566.

28. Biever, A., et al., Monosomes actively translate synaptic mRNAs in neuronal processes. Science, 2020. 367(6477).

29. Terenzio, M., et al., Locally translated mTOR controls axonal local translation in nerve injury. Science, 2018. 359(6382): p. 1416-1421.

30. Leung, K.M., et al., Asymmetrical beta-actin mRNA translation in growth cones mediates attractive turning to netrin-1. Nat Neurosci, 2006. 9(10): p. 1247-56.

31. Santos, A.R., D. Comprido, and C.B. Duarte, Regulation of local translation at the synapse by BDNF. Prog Neurobiol, 2010. 92(4): p. 505-16.

32. Rathod, R., et al., Laminin induced local axonal translation of beta-actin mRNA is impaired in SMNdeficient motoneurons. Histochem Cell Biol, 2012. 138(5): p. 737-48.

33. Sasaki, Y., et al., Phosphorylation of zipcode binding protein 1 is required for brain-derived neurotrophic factor signaling of local beta-actin synthesis and growth cone turning. J Neurosci, 2010. 30(28): p. 9349-58.

34. Huang, E.J. and L.F. Reichardt, Trk receptors: roles in neuronal signal transduction. Annu Rev Biochem, 2003. 72: p. 609-42.

35. Leal, G., D. Comprido, and C.B. Duarte, BDNF-induced local protein synthesis and synaptic plasticity. Neuropharmacology, 2014. 76 Pt C: p. 639-56.

36. Willis, D.E., et al., Extracellular stimuli specifically regulate localized levels of individual neuronal mRNAs. J Cell Biol, 2007. 178(6): p. 965-80. 
37. Yoon, B.C., K.H. Zivraj, and C.E. Holt, Local translation and mRNA trafficking in axon pathfinding. Results Probl Cell Differ, 2009. 48: p. 269-88.

38. Maday, S., et al., Axonal transport: cargo-specific mechanisms of motility and regulation. Neuron, 2014. 84(2): p. 292-309.

39. Ben-Shem, A., et al., The structure of the eukaryotic ribosome at 3.0 A resolution. Science, 2011. 334(6062): p. 1524-9.

40. Tsai, N.P., et al., Netrin-1 signaling regulates de novo protein synthesis of kappa opioid receptor by facilitating polysomal partition of its mRNA. J Neurosci, 2006. 26(38): p. 9743-9.

41. Willis, D., et al., Differential transport and local translation of cytoskeletal, injury-response, and neurodegeneration protein mRNAs in axons. J Neurosci, 2005. 25(4): p. 778-91.

42. Briese, M., et al., Loss of Tdp-43 disrupts the axonal transcriptome of motoneurons accompanied by impaired axonal translation and mitochondria function. Acta Neuropathol Commun, 2020. 8(1): p. 116.

43. Costa, C.J. and D.E. Willis, To the end of the line: Axonal mRNA transport and local translation in health and neurodegenerative disease. Dev Neurobiol, 2018. 78(3): p. 209-220.

44. De Gregorio, C., et al., Drosophila Atlastin in motor neurons is required for locomotion and presynaptic function. J Cell Sci, 2017. 130(20): p. 3507-3516.

45. Summerville, J.B., et al., The effects of ER morphology on synaptic structure and function in Drosophila melanogaster. J Cell Sci, 2016. 129(8): p. 1635-48.

46. Lindhout, F.W., et al., VAP-SCRN1 interaction regulates dynamic endoplasmic reticulum remodeling and presynaptic function. EMBO J, 2019. 38(20): p. e101345.

47. Friedman, J.R., et al., ER sliding dynamics and ER-mitochondrial contacts occur on acetylated microtubules. J Cell Biol, 2010. 190(3): p. 363-75.

48. Terasaki, M., L.B. Chen, and K. Fujiwara, Microtubules and the endoplasmic reticulum are highly interdependent structures. J Cell Biol, 1986. 103(4): p. 1557-68.

49. Dreier, L. and T.A. Rapoport, In vitro formation of the endoplasmic reticulum occurs independently of microtubules by a controlled fusion reaction. J Cell Biol, 2000. 148(5): p. 883-98.

50. Wang, S., et al., Multiple mechanisms determine ER network morphology during the cell cycle in Xenopus egg extracts. J Cell Biol, 2013. 203(5): p. 801-14.

51. Voeltz, G.K., et al., A class of membrane proteins shaping the tubular endoplasmic reticulum. Cell, 2006. 124(3): p. 573-86.

52. Wang, Y., M.P. Mattson, and K. Furukawa, Endoplasmic reticulum calcium release is modulated by actin polymerization. J Neurochem, 2002. 82(4): p. 945-52.

53. Dent, E.W. and F.B. Gertler, Cytoskeletal dynamics and transport in growth cone motility and axon guidance. Neuron, 2003. 40(2): p. 209-27.

54. Dent, E.W., S.L. Gupton, and F.B. Gertler, The growth cone cytoskeleton in axon outgrowth and guidance. Cold Spring Harb Perspect Biol, 2011. 3(3). 
55. Mallavarapu, A. and T. Mitchison, Regulated actin cytoskeleton assembly at filopodium tips controls their extension and retraction. J Cell Biol, 1999. 146(5): p. 1097-106.

56. Dogterom, M. and G.H. Koenderink, Actin-microtubule crosstalk in cell biology. Nat Rev Mol Cell Biol, 2019. 20(1): p. 38-54.

57. Meka, D.P., et al., Radial somatic F-actin organization affects growth cone dynamics during early neuronal development. EMBO Rep, 2019. 20(12): p. e47743.

58. Zhao, B., et al., Microtubules Modulate F-actin Dynamics during Neuronal Polarization. Sci Rep, 2017. 7(1): p. 9583.

59. Boutry, M., S. Morais, and G. Stevanin, Update on the Genetics of Spastic Paraplegias. Curr Neurol Neurosci Rep, 2019. 19(4): p. 18.

60. Park, S.H., et al., Hereditary spastic paraplegia proteins REEP1, spastin, and atlastin-1 coordinate microtubule interactions with the tubular ER network. J Clin Invest, 2010. 120(4): p. 1097-110.

61. Ridge, P.G., et al., Correction to: Linkage, whole genome sequence, and biological data implicate variants in RAB10 in Alzheimer's disease resilience. Genome Med, 2018. 10(1): p. 4.

62. Shim, S.Y., et al., Protein 600 is a microtubule/endoplasmic reticulum-associated protein in CNS neurons. J Neurosci, 2008. 28(14): p. 3604-14.

63. Nishimura, A.L., et al., A mutation in the vesicle-trafficking protein VAPB causes late-onset spinal muscular atrophy and amyotrophic lateral sclerosis. Am J Hum Genet, 2004. 75(5): p. 822-31.

64. Hensel, N. and P. Claus, The Actin Cytoskeleton in SMA and ALS: How Does It Contribute to Motoneuron Degeneration? Neuroscientist, 2018. 24(1): p. 54-72.

65. Giesemann, T., et al., Complex formation between the postsynaptic scaffolding protein gephyrin, profilin, and Mena: a possible link to the microfilament system. J Neurosci, 2003. 23(23): p. 8330-9.

66. Nolle, A., et al., The spinal muscular atrophy disease protein SMN is linked to the Rho-kinase pathway via profilin. Hum Mol Genet, 2011. 20(24): p. 4865-78.

67. Sharma, A., et al., A role for complexes of survival of motor neurons (SMN) protein with gemins and profilin in neurite-like cytoplasmic extensions of cultured nerve cells. Exp Cell Res, 2005. 309(1): p. 185-97.

68. Oprea, G.E., et al., Plastin 3 is a protective modifier of autosomal recessive spinal muscular atrophy. Science, 2008. 320(5875): p. 524-7.

69. Lyon, A.N., et al., Calcium binding is essential for plastin 3 function in Smn-deficient motoneurons. Hum Mol Genet, 2014. 23(8): p. 1990-2004.

70. Torres-Benito, L., et al., SMN requirement for synaptic vesicle, active zone and microtubule postnatal organization in motor nerve terminals. PLoS One, 2011. 6(10): p. e26164.

71. Poulopoulos, A., et al., Subcellular transcriptomes and proteomes of developing axon projections in the cerebral cortex. Nature, 2019. 565(7739): p. 356-360.

72. Ming, G.L., et al., CAMP-dependent growth cone guidance by netrin-1. Neuron, 1997. 19(6): p. 122535 . 
73. Bernabo, P., et al., In Vivo Translatome Profiling in Spinal Muscular Atrophy Reveals a Role for SMN Protein in Ribosome Biology. Cell Rep, 2017. 21(4): p. 953-965.

74. Sanchez, G., et al., A novel function for the survival motoneuron protein as a translational regulator. Hum Mol Genet, 2013. 22(4): p. 668-84.

75. Lauria, F., et al., SMN-primed ribosomes modulate the translation of transcripts related to spinal muscular atrophy. Nat Cell Biol, 2020. 22(10): p. 1239-1251.

76. Bechade, C., et al., Subcellular distribution of survival motor neuron (SMN) protein: possible involvement in nucleocytoplasmic and dendritic transport. Eur J Neurosci, 1999. 11(1): p. 293-304.

77. Chai, $\mathrm{H}$., et al., Long-term effects of a single dose of brain-derived neurotrophic factor on motoneuron survival following spinal root avulsion in the adult rat. Neurosci Lett, 1999. 274(3): p. 147-50.

78. McKay, S.E., et al., The expression of trkB and $p 75$ and the role of BDNF in the developing neuromuscular system of the chick embryo. Development, 1996. 122(2): p. 715-24.

79. Sendtner, M., et al., Brain-derived neurotrophic factor prevents the death of motoneurons in newborn rats after nerve section. Nature, 1992. 360(6406): p. 757-9.

80. Andreska, T., P. Luningschror, and M. Sendtner, Regulation of TrkB cell surface expression-a mechanism for modulation of neuronal responsiveness to brain-derived neurotrophic factor. Cell Tissue Res, 2020. 382(1): p. 5-14.

81. Zhao, L., et al., Mechanism underlying activity-dependent insertion of TrkB into the neuronal surface. J Cell Sci, 2009. 122(Pt 17): p. 3123-36.

82. Monani, U.R., et al., The human centromeric survival motor neuron gene (SMN2) rescues embryonic lethality in Smn-/- mice and results in a mouse with spinal muscular atrophy. Human Molecular Genetics, 2000. 9(3): p. 333-339.

83. Rohrer, B., et al., Role of neurotrophin receptor TrkB in the maturation of rod photoreceptors and establishment of synaptic transmission to the inner retina. J Neurosci, 1999. 19(20): p. 8919-30.

84. Wiese, S., et al., Isolation and enrichment of embryonic mouse motoneurons from the lumbar spinal cord of individual mouse embryos. Nature Protocols, 2009. 5(1): p. 31-38.

85. Fox, M.A., et al., Distinct target-derived signals organize formation, maturation, and maintenance of motor nerve terminals. Cell, 2007. 129(1): p. 179-93.

86. Rogers, R.S. and H. Nishimune, The role of laminins in the organization and function of neuromuscular junctions. Matrix Biol, 2017. 57-58: p. 86-105.

87. Sivadasan, R., et al., C9ORF72 interaction with cofilin modulates actin dynamics in motor neurons. Nat Neurosci, 2016. 19(12): p. 1610-1618.

88. Subramanian, N., et al., Role of Nav1.9 in activity-dependent axon growth in motoneurons. Human Molecular Genetics, 2012. 21(16): p. 3655-3667.

\section{Figures}



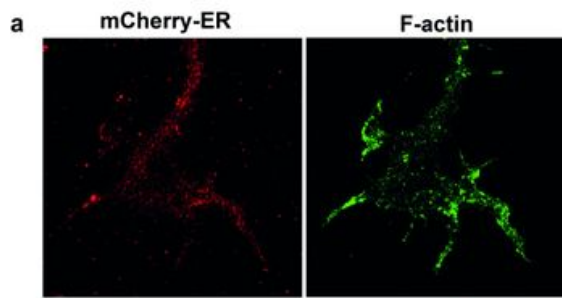

a-Tubulin Merge
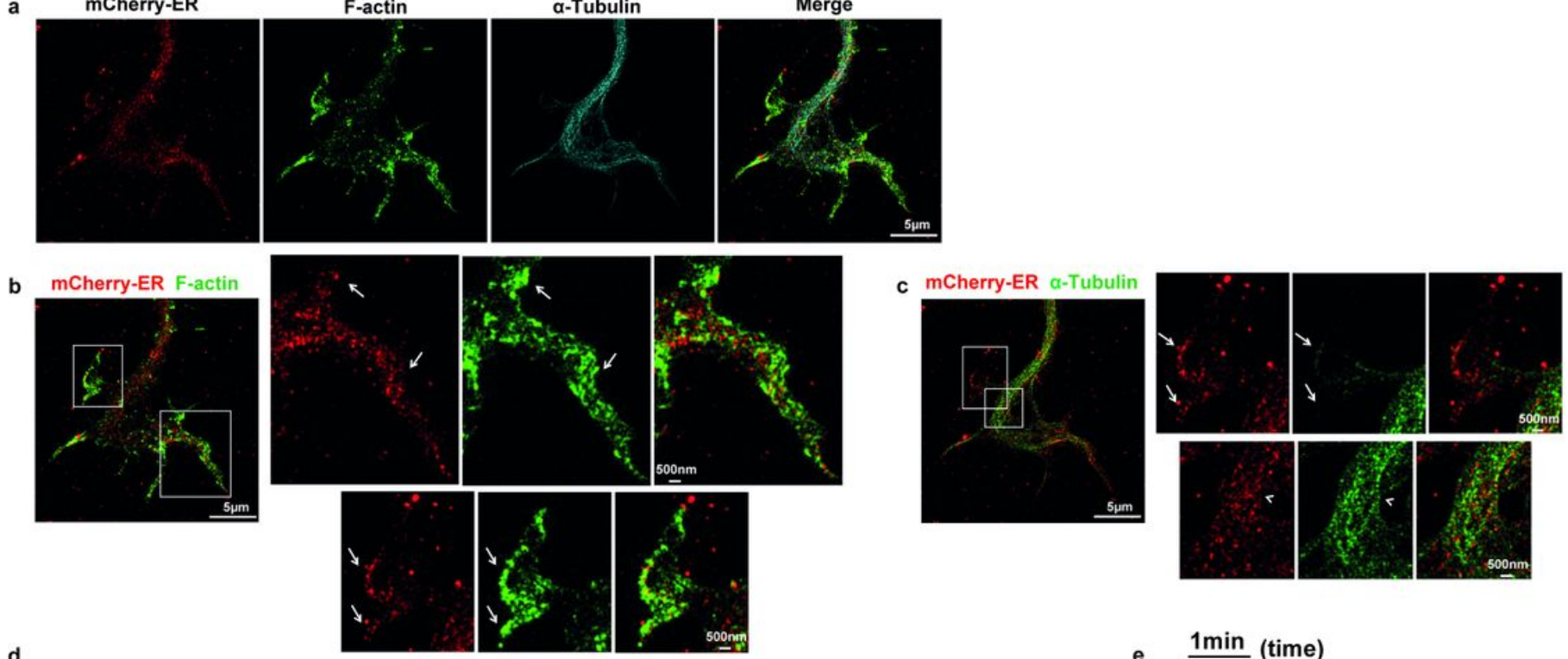

c

문
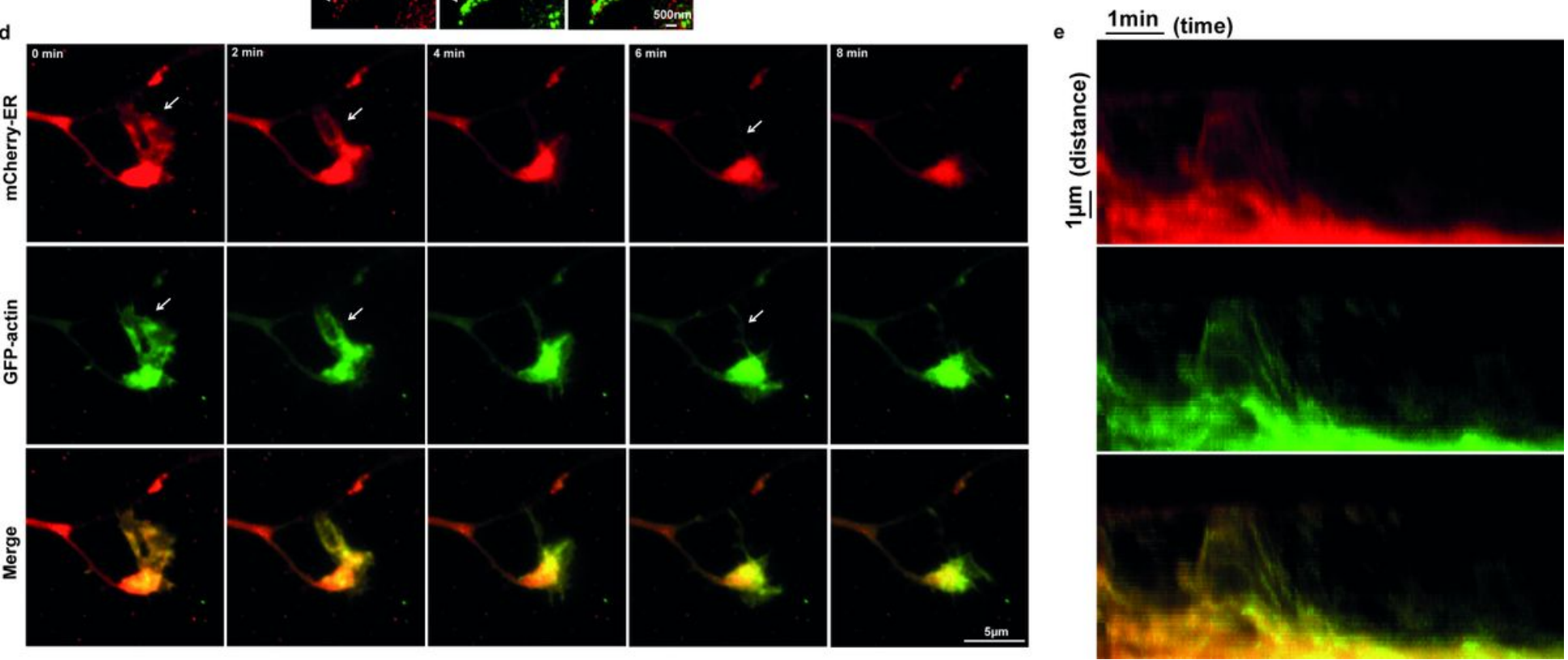

\section{Figure 1}

ER is present in growth cones and moves along actin filaments in growth cone filopodia. (a) Motoneurons were transduced with a lentiviral construct expressing mCherry-KDEL (mCherry-ER) to visualize ER in the growth cone. To determine growth cone boundaries, motoneurons were immunostained against F-actin and a-Tubulin in addition to mCherry. Images captured by SIM show that mCherry-ER is present in both core and filopodia sub-regions of the growth cone. (b) Representative overlap image of mCherry-ER (red) and F-actin (green). White square boxes indicate magnification of ROls in filopodia. Arrows indicate overlapping of mCherry-ER and F-actin in filopodia. (c) Representative overlap image of mCherry-ER (red) and a-Tubulin (green). White square boxes indicate magnification of ROls in filopodia and core. Arrows indicate that mCherry-ER does not overlap with a-Tubulin in filopodia. Arrowheads show mCherry-ER and a-Tubulin overlap in the growth cone core. (d) Representative time-lapse images of motoneurons transduced with a lentivirus co-expressing GFP-actin and mCherry-KDEL. Arrows indicate that mCherry-ER 
moves along with GFP-actin in filopodia. (e) Multiple kymographs show ER (red) and actin co-movements (green) in filopodia.

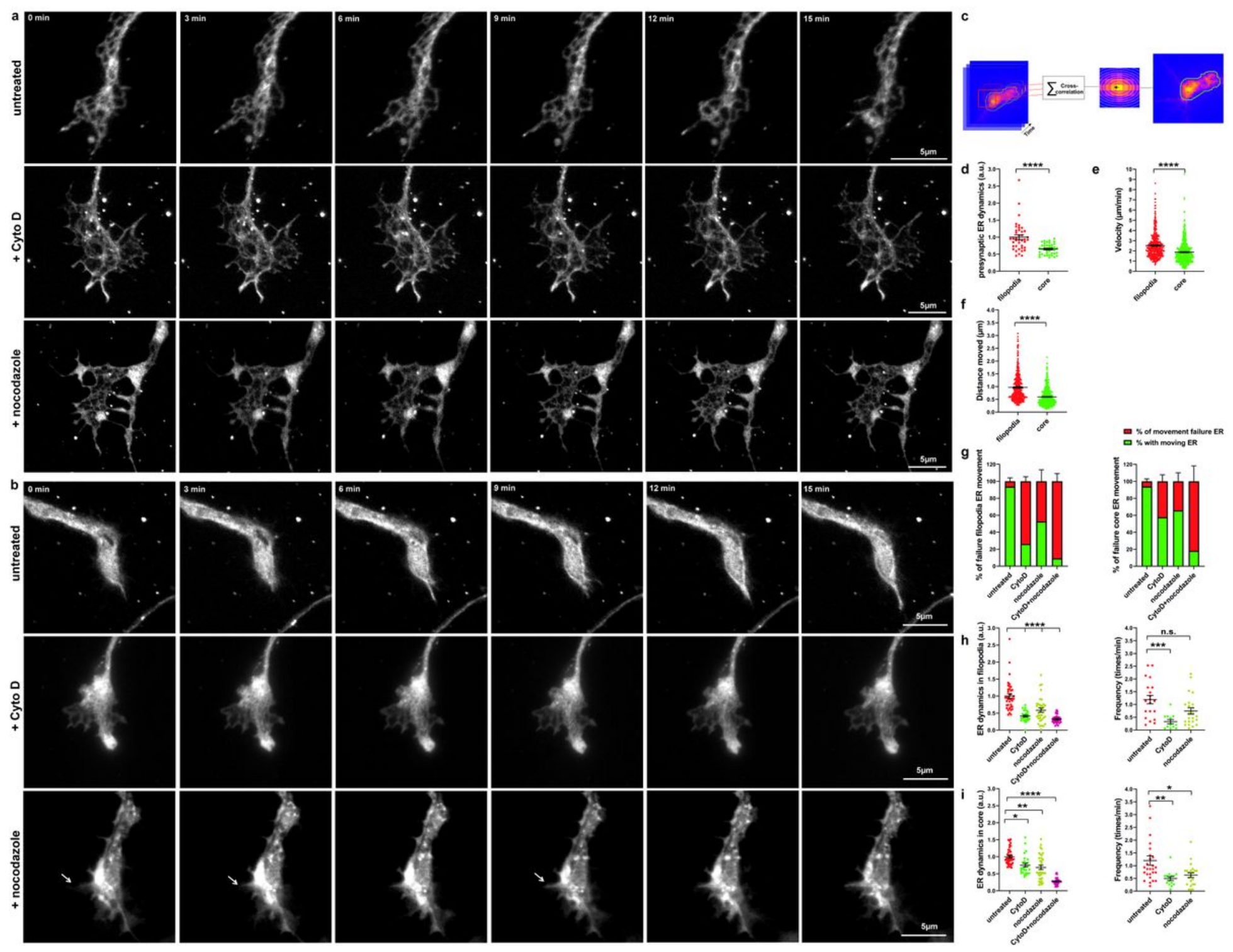

Figure 2

ER dynamic movements are regulated by actin and microtubules in growth cone core and filopodia. (a and b) Representative time-lapse images of motoneurons expressing mCherry-ER in the growth cone core (a) and filopodia (b). Arrows show ER extension into filopodia. (c) Diagram shows workflow of ICS python implementation. (d) Graph shows ICS-analysis of ER movements in filopodia versus core. ER dynamic movements are higher in filopodia versus core $(\star \star \star \star, P<0.0001 ; n=40$ cells from 6 independent experiments). (e and f) Kymograph-analysis of velocity of ER movements (e) and distance moved by ER (f) in filopodia versus core ( $* \star \star \star, P<0.0001 ; n=25-29$ cells from 3 independent experiments). (g) Average percentage of neurons failing to show ER movements in filopodia or core upon treatments $(n=32$ cells from 3 independent experiments). (h) Average ER movements (left panel: $* \star \star \star, ~ P<0.0001 ; n=27-40$ cells from 4-5 independent experiments) and frequency of ER movements (right panel) in filopodia per growth cone. In filopodia, frequency of ER movements is decreased upon CytoD ( $* \star \star, ~ P=0.0004 ; n=12-20$ cells) 
but not nocodazole treatment (n.s., $P=0.1079 ; n=20-23$ cells from 4-5 independent experiments).

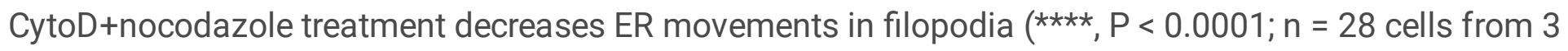
independent experiments). (i) Average ER movements (left panel: ${ }^{*}, P=0.0353 ; * \star, P=0.0012 ; n=27-40$ cells from 4-5 independent experiments) and frequency of ER movements (right panel: **, $P=0.0016 ; *, P$ $=0.0271 ; n=15-27$ cells from 4-6 independent experiments) in core per growth cone. CytoD+nocodazole treatment decreases core ER movements ( $* \star \star \star, P<0.0001 ; n=28-40$ cells from 3 independent experiments). Graphs are shown in scatter dot plot with mean \pm SEM. Statistical analyses: One-way ANOVA with Dunn's post-test in $\mathrm{h}$ and $\mathrm{i}$, and Mann Whitney test in $\mathrm{d}-\mathrm{f}$.
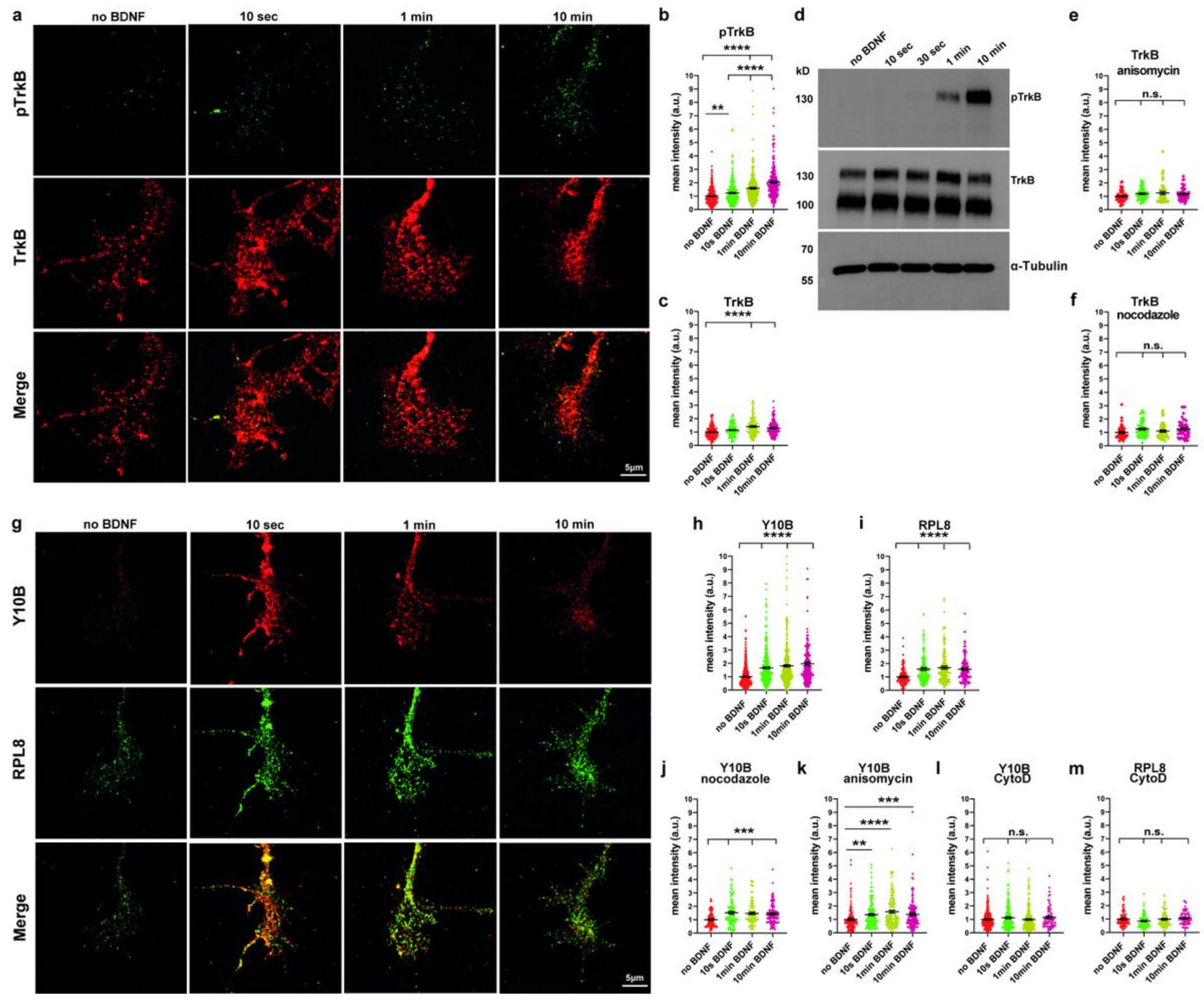

Figure 3

BDNF-induced TrkB activation triggers redistribution of ribosomes in growth cones. (a) Representative images of growth cones of BDNF-stimulated motoneurons stained against pTrkB and TrkB. (b and $c$ ) Mean intensities of $p$ TrkB increase at $10 \mathrm{sec}(* *, P=0.0063 ; n=118-267$ cells) and mean intensities of pTrkB and TrkB increase at $1 \mathrm{~min}(* \star * *, P<0.0001 ; \mathrm{n}=123-274$ cells) and 10 min poststimulation (****, $\mathrm{P}$ 
$<0.0001 ; n=120-266$ cells from 8 and 4 independent experiments). (d) Representative immunoblot of total lysates from cultured motoneurons probed against pTrkB, TrkB and a-Tubulin. (e and f) Total levels of TrkB in stimulated growth cones treated with nocodazole (n.s., $P \geq 0.1526 ; n=45-46$ cells) or anisomycin (n.s., $P \geq 0.0893 ; n=47-54$ cells from 2 independent experiments). (g) Representative images of stimulated growth cones stained against Y10B and RPL8. ( $h$ and i) Mean intensities of Y10B and RPL8 increase at $10 \mathrm{sec}\left({ }^{\star * \star *}, \mathrm{P}<0.0001 ; \mathrm{n}=138-366\right.$ cells), $1 \mathrm{~min}(* \star \star \star, P<0.0001 ; \mathrm{n}=138-366$ cells from 9 and 4 independent experiments) and 10 min poststimulation ( $\star \star \star \star, P<0.0001 ; n=138-366$ cells from 6

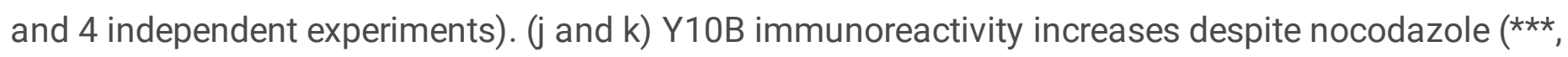
$0.0002 \leq P \leq 0.0004 ; n=93-102$ cells from 3 independent experiments) or anisomycin treatments (**, $P$ $=0.0012 ; * \star \star \star, P<0.0001 ; * \star \star, P=0.0008 ; \mathrm{n}=121-141$ cells from 5 independent experiments). ( $\mathrm{l}$ and $\mathrm{m})$ $\mathrm{Y} 10 \mathrm{~B}$ (n.s., $\mathrm{P} \geq 0.5207, \mathrm{n}=68-252$ cells from $3-6$ independent experiments) and RPL8 immunoreactivities (n.s., $P \geq 0.2256, n=68-87$ cells from 3 independent experiments) do not increase with CytoD treatment. All data are normalized to the average intensities of no BDNF group. Graphs are shown in scatter dot plot with mean \pm SEM. Statistical analyses: One-way ANOVA with Dunn's post-test. 


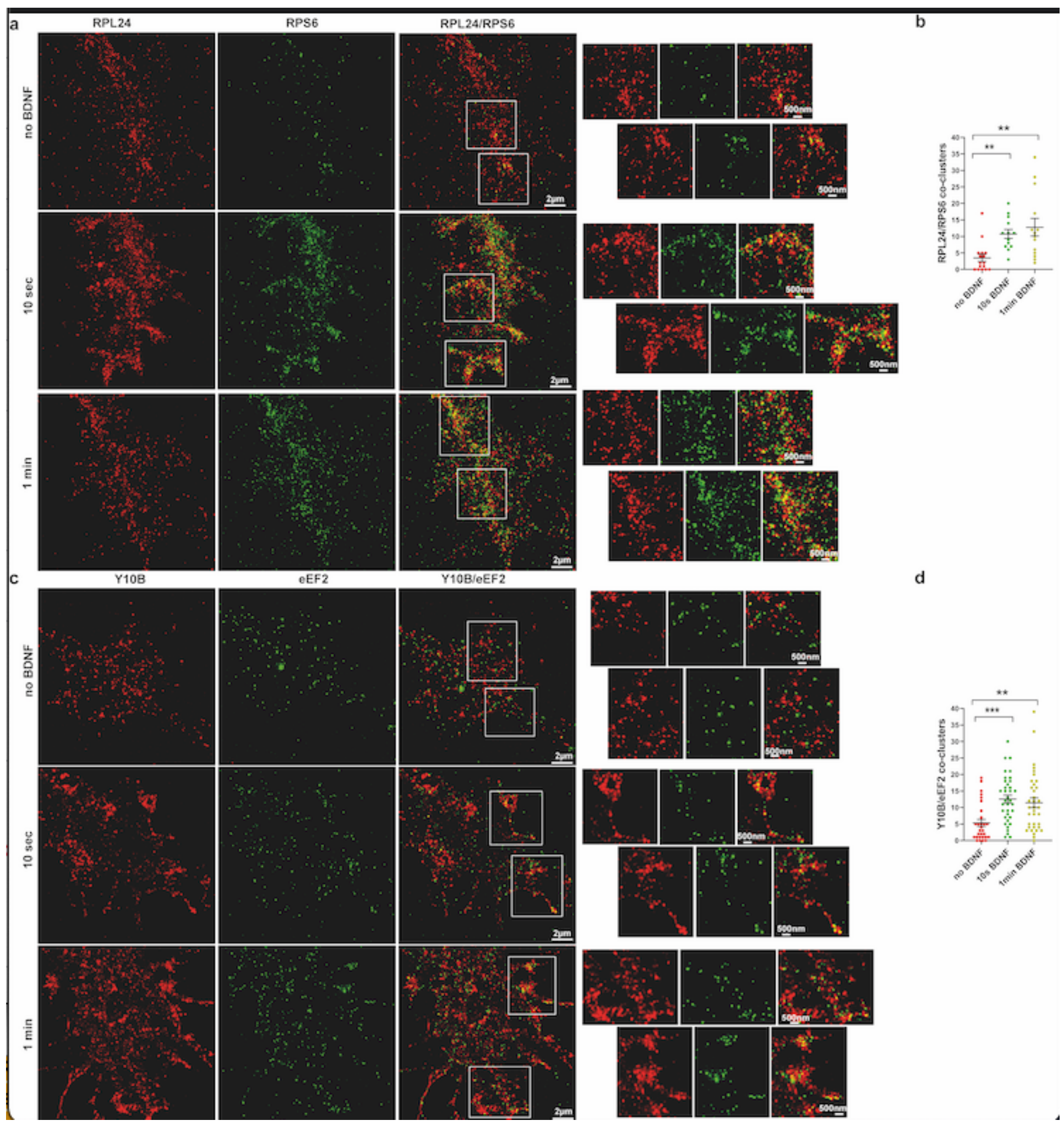

Figure 4

BDNF stimulation induces ribosomal assembly and initiates translation in growth cones of motoneurons.

(a) Representative SIM images of growth cones of BDNF-stimulated Smn+/+; SMN2tgtg motoneurons stained against RPL24 and RPS6. White squares indicate magnification of ROIs in growth cones. BDNF stimulation induces formation of RPL24/RPS6 co-clusters at $10 \mathrm{sec}$ and $1 \mathrm{~min}$. For a better comparability to Fig. 9, Smn+/+; SMN2tgtg motoneurons were used as WT. (b) Quantification of RPL24/RPS6 co- 
clusters that are representative of fully assembled $80 S$ subunits $(* \star, 0.0016 \leq P \leq 0.0019 ; n=13-16$ cells from 3 independent experiments). (c) Representative SIM images of growth cones of BDNF-stimulated motoneurons stained against $\mathrm{Y} 10 \mathrm{~B}$ and eEF2. White squares indicate magnification of ROIs in growth cones. BDNF stimulation induces formation of Y10B/eEF2 co-clusters at $10 \mathrm{sec}$ as well as $1 \mathrm{~min}$. (d) Quantification of $\mathrm{Y} 10 \mathrm{~B} / \mathrm{eEF} 2$ co-clusters that are representative of ribosomes in the elongation phase of translation ( $* \star \star, P=0.0001 ; * \star, P=0.0037 ; n=29-36$ cells from 3 independent experiments). Graphs are shown in scatter dot plot with mean \pm SEM. Statistical analyses: One-way ANOVA with Dunn's post-test.

a
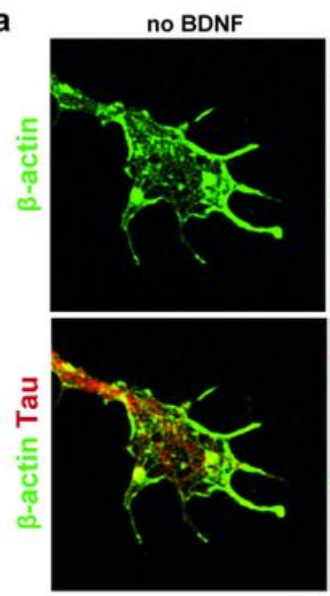

e

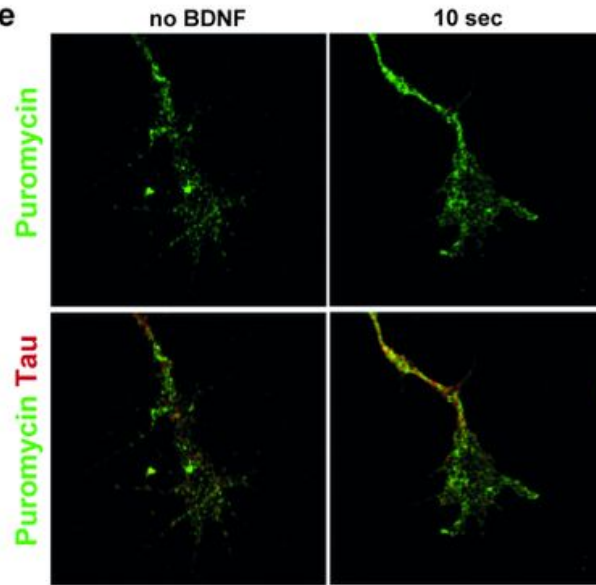

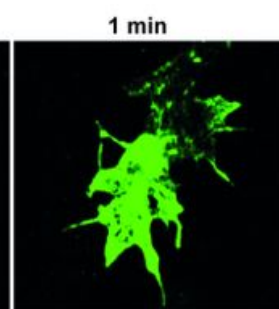
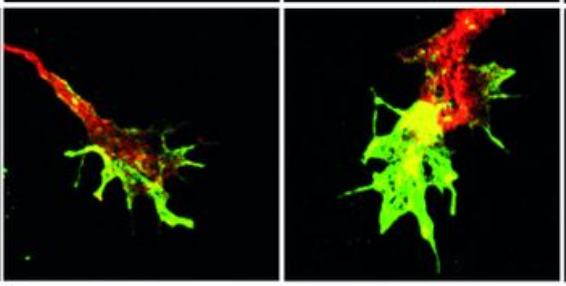

$1 \mathrm{~min}$

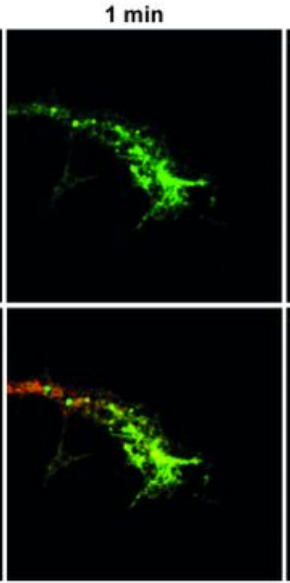

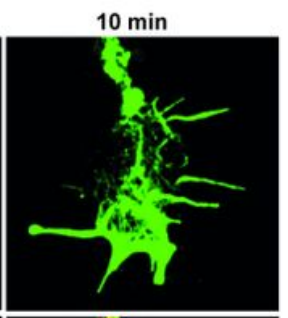
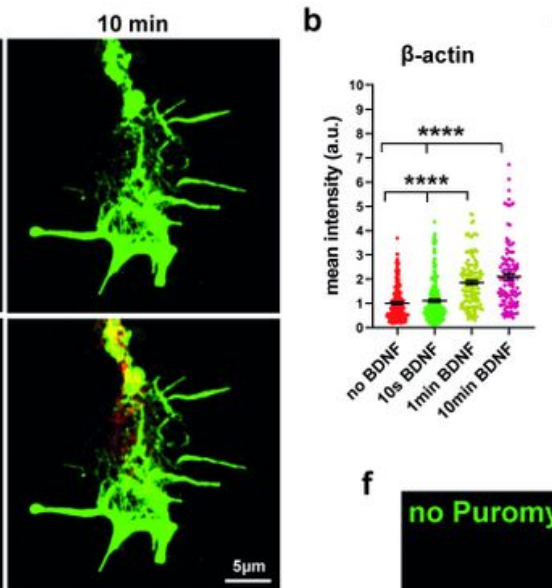

c

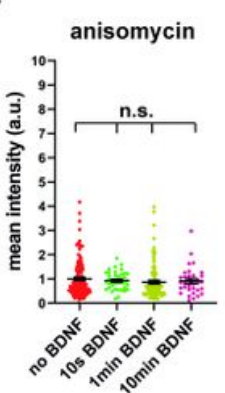

f

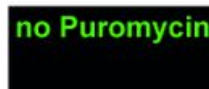

$10 \mathrm{~min}$
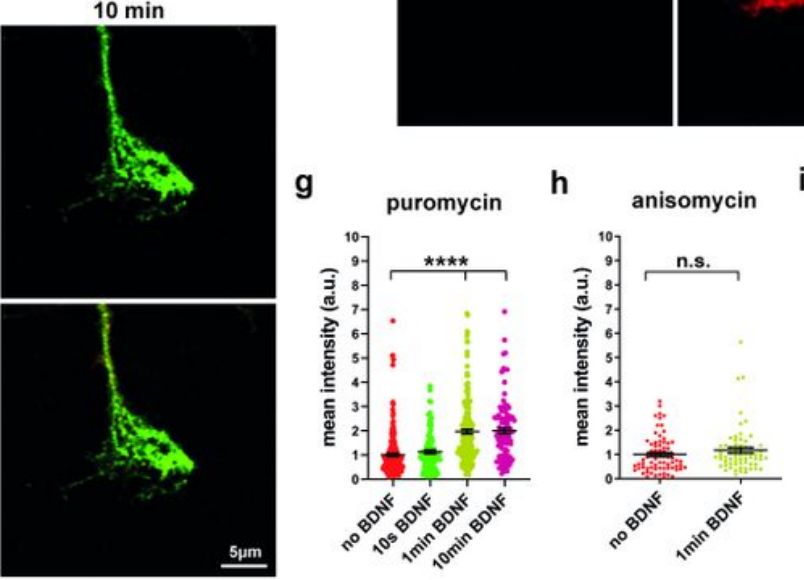

d

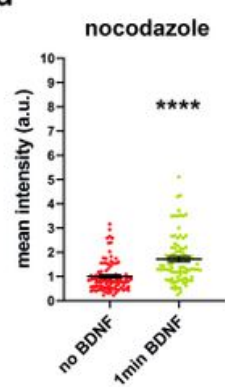

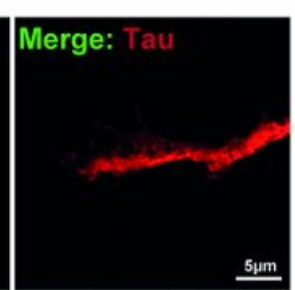

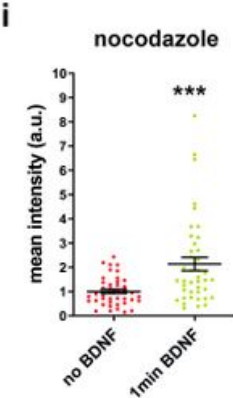

\section{Figure 5}

Rapid effect of BDNF stimulation on protein synthesis in motoneuron growth cones. (a) Representative confocal images of growth cones of BDNF-stimulated motoneurons stained against $\beta$-actin (green) and Tau (red). (b) Graph shows mean intensities of $\beta$-actin in growth cones. A substantial enhancement in mean intensity of $\beta$-actin immunoreactivity is first detected after 1 min or 10 min stimulation ( $* \star \star \star, P<$ 0.0001; $n=114-202$ cells from 4 independent experiments). (c) Anisomycin-treated cells do not show increased levels of $\beta$-actin in the growth cone in response to BDNF stimulation ( $n$.S., $P \geq 0.2903 ; n=33$ 127 cells from 2-3 independent experiments). (d) 1 min BDNF stimulation leads to an increase in $\beta$-actin protein levels in nocodazole-treated cells ( $\star \star \star \star, P<0.0001 ; n=91-95$ cells from 3 independent experiments). (e) Representative confocal images of growth cones of BDNF-stimulated motoneurons stained against puromycin (green) and Tau (red). (f) As control, puromycin was omitted and cells were incubated only with primary and secondary antibodies against puromycin. (g) Puromycin 
immunoreactivity levels are increased at $1 \mathrm{~min}$ and $10 \mathrm{~min}$ post-stimulation ( $\star \star \star \star, ~ P<0.0001 ; n=103-218$ cells from 3-6 independent experiments). (h and i) Anisomycin (n.s., $P=0.1796 ; n=65-73$ cells from 2 independent experiments) but not nocodazole ( $* \star *, P=0.0002 ; n=42$ cells from 2 independent experiments) treatment inhibits puromycin immunoreactivity after BDNF stimulation. All data are normalized to the average intensities of no BDNF treatment group. Graphs are shown in scatter dot plot with mean \pm SEM. Statistical analyses: One-way ANOVA with Dunn's post-test in b, c and g, and Mann Whitney test in $\mathrm{d}, \mathrm{h}$ and $\mathrm{i}$.
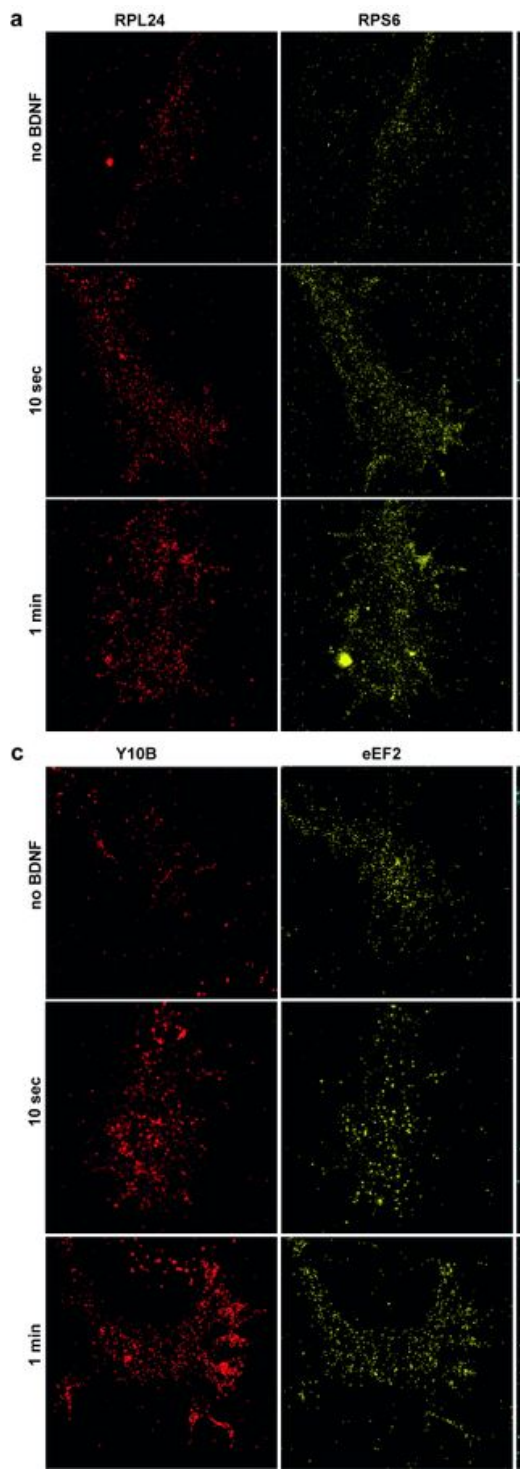

mCherry-ER

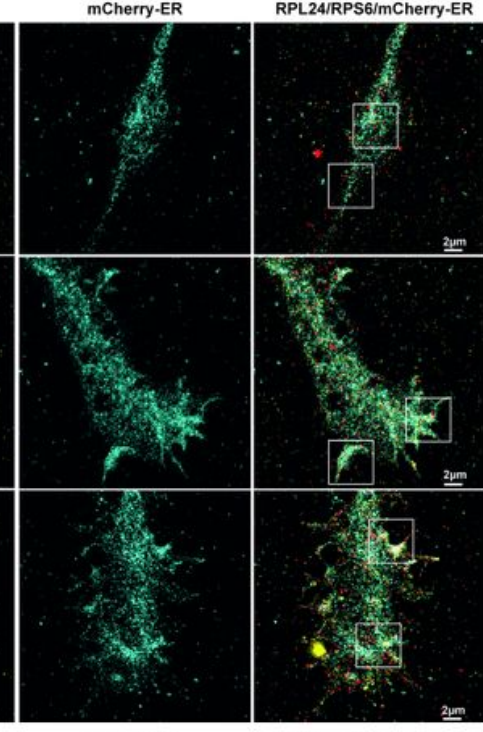

mCherry-ER

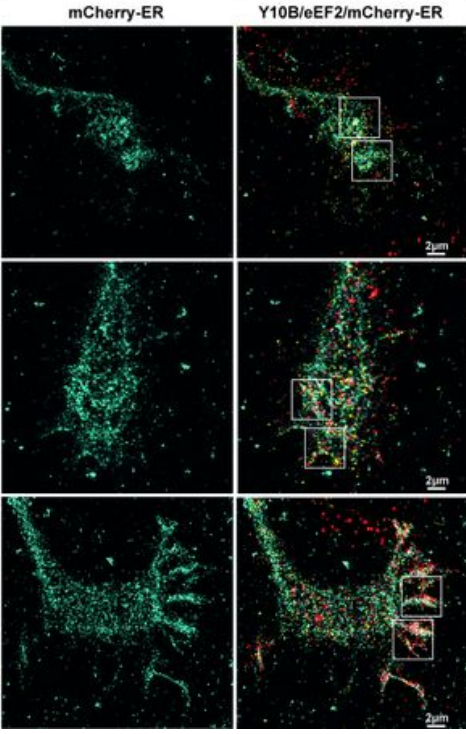

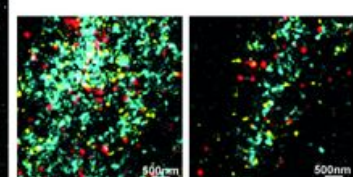
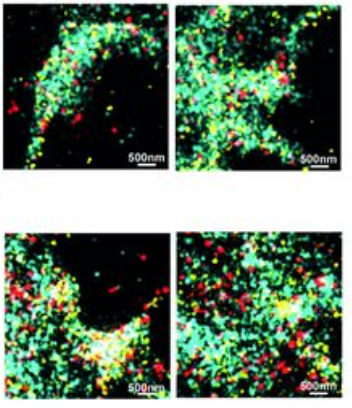

b

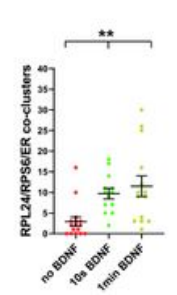

d
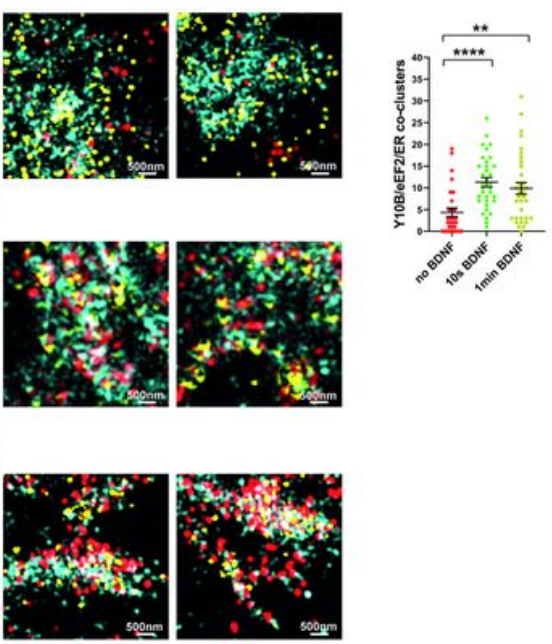

e
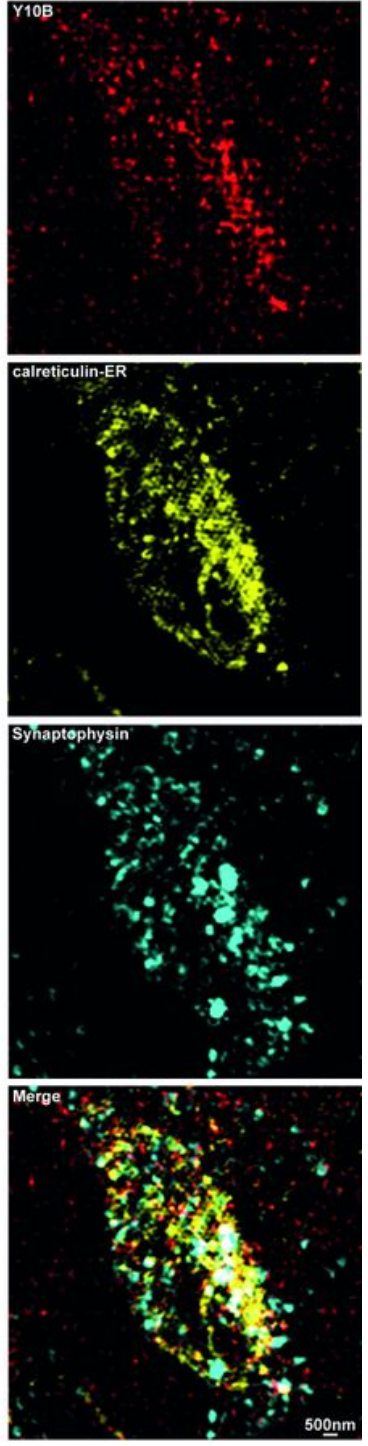

Figure 6

Ribosomes attach to the presynaptic ER and promote local translation in response to BDNF stimulation. (a) BDNF-stimulated motoneurons expressing mCherry-ER were stained against RPL24 and RPS6, and growth cones were imaged by SIM. For a better comparability to Fig. 9, Smn+/+; SMN2tgtg motoneurons were used as WT. (b) Graph shows co-cluster formation of RPL24/RPS6 with ER within $10 \mathrm{sec}$ and 1 min of stimulation ( ${ }^{*}, 0.0015 \leq \mathrm{P} \leq 0.0026 ; n=13-16$ cells from 3 independent experiments). (c) BDNFstimulated motoneurons expressing mCherry-ER were stained against $\mathrm{Y} 10 \mathrm{~B}$ and eEF2 and growth cones were imaged by SIM. (d) Graph shows co-cluster formation of Y10B/eEF2 with ER within 10 sec and 1 
min stimulation ( $\star \star \star \star, P<0.0001 ; * \star, P=0.0029 ; n=29-34$ cells from 3 independent experiments). (e) TVA muscles were dissected from P5 mice and NMJs were immunostained with antibodies against $\mathrm{Y} 10 \mathrm{~B}$ and calreticulin. Synaptophysin staining was used to label presynaptic terminals. Representative images captured by SIM show the colocalization of Y10B and calreticulin in synaptophysin positive NMJs. Graphs are shown in scatter dot plot with mean \pm SEM. Statistical analyses: One-way ANOVA with Dunn's post-test.
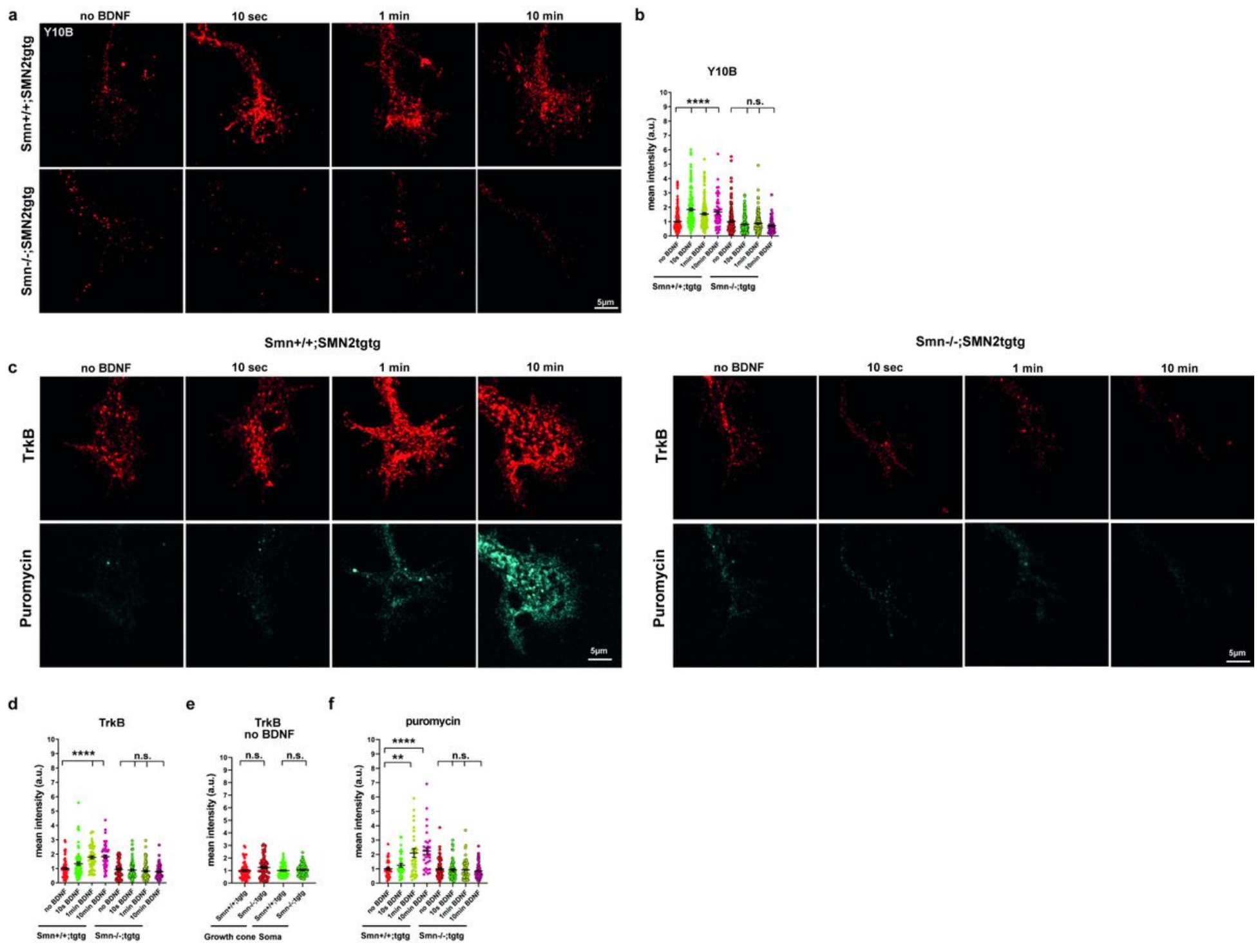

\section{Figure 7}

Smn-deficient motoneurons exhibit impaired BDNF/TrkB responses in growth cones. (a) Representative images of growth cones of motoneurons isolated from WT and Smn-deficient mice immunostained using Y10B antibody. (b) Quantification of Y10B mean intensities shows that in contrast to Smn+/+SMN2tgtg

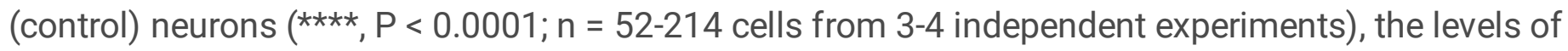
Y10B do not increase in growth cones of BDNF-stimulated Smn-deficient motoneurons (n.s., P $\geq 0.0774$; $n=51-202$ cells from 3-5 independent experiments). (c) Representative images of growth cones of Smndeficient and control motoneurons immunostained against TrkB and puromycin. (d) Quantification of TrkB mean intensities in growth cones of BDNF-stimulated control and Smn-deficient motoneurons 
indicates no increase in TrkB levels in response to BDNF pulse in Smn-deficient neurons (n.S., $\mathrm{P} \geq 0.6078$;

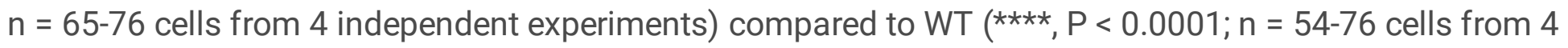
independent experiments). (e) Quantification of TrkB mean intensities in control and Smn-deficient neurons under no stimulation condition in growth cones and soma (n.s., $P=0.0512 ; n$.s., $P=0.4546 ; n=$ 71-85 cells from 4 independent experiments). (f) Puromycin immunoreactivity does not increase in BDNFstimulated Smn-deficient neurons (n.s., $\mathrm{P}>$ 0.9999; $\mathrm{n}=61-68$ cells from 3 independent experiments) compared to control ( $* *, P=0.0061 ; * \star \star \star, ~ P<0.0001 ; n=32-83$ cells from 2 independent experiments). In e, all data are normalized to control. In pulse experiments, data are normalized to no BDNF group of the corresponding genotype. Graphs are shown in scatter dot plot with mean \pm SEM. Statistical analyses: One-way ANOVA with Dunn's post-test.

a
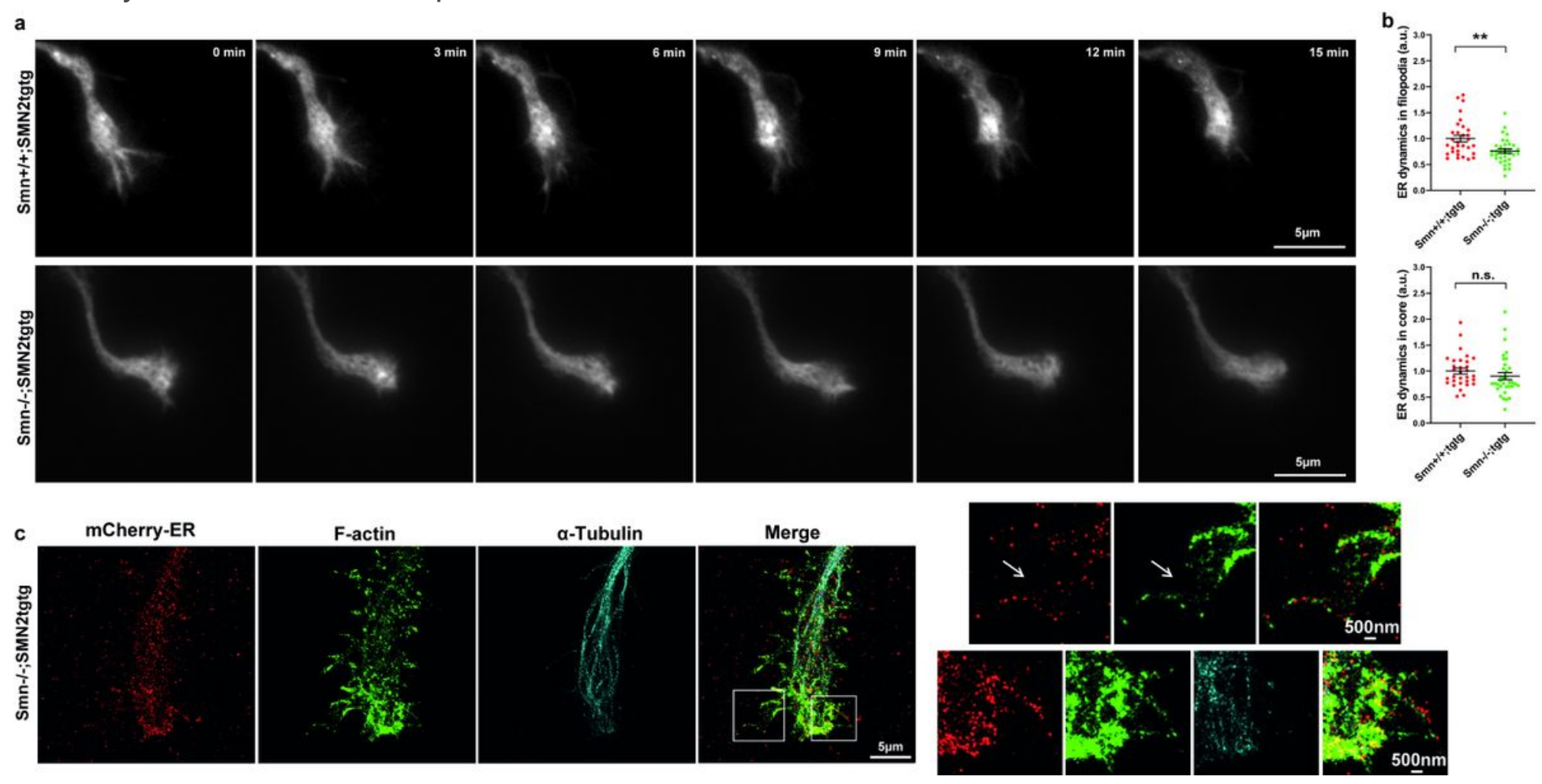

\section{Figure 8}

ER dynamic movements in growth cone filopodia are disturbed in Smn-deficient motoneurons. (a) Representative time-lapse images of Smn+/+SMN2tgtg (control) and Smn-deficient motoneurons expressing mCherry-ER in growth cones. (b) Dynamics of ER movements were analyzed in growth cone filopodia and core. ER dynamic movements in filopodia ( $* \star, P=0.004 ; n=34$ cells from 3 independent experiments) but not in the core (n.s., $\mathrm{P}=0.0708 ; \mathrm{n}=31-24$ cells from 3 independent experiments) are lower in Smn-deficient motoneurons compared to control. All data are normalized to Smn+/+SMN2tgtg control. (c) Motoneurons from Smn-deficient mice were transduced with lentivirus expressing mCherryKDEL and immunostained against mCherry-ER, F-actin and a-Tubulin. Representative SIM images of growth cones show a deficiency in ER entry into filopodia that correlates with defective F-actin organization in filopodia, indicated by arrows. The inserts enlarge images marked by white squares. Graphs are shown in scatter dot plot with mean \pm SEM. Statistical analyses: Mann Whitney test. 


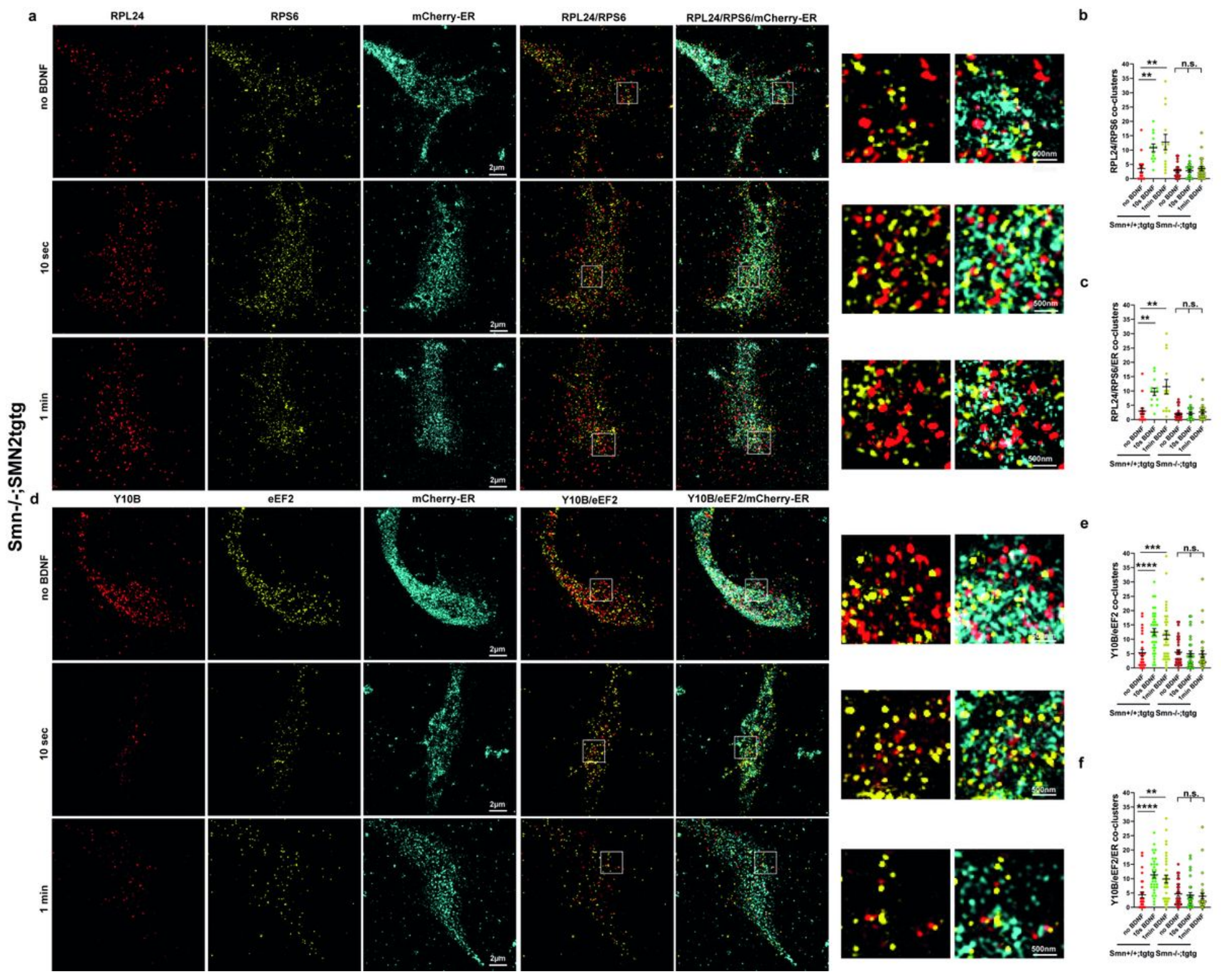

Figure 9

BDNF/TrkB induced local translation is disturbed in growth cones of Smn-deficient motoneurons. (a) BDNF-stimulated Smn-deficient motoneurons expressing mCherry-ER were stained against RPL24 and RPS6 and growth cones were imaged by SIM. White boxes indicate enlarged ROls within growth cones. (b) In contrast to Smn+/+; SMN2tgtg, Smn-deficient motoneurons do not exhibit increased number of RPL24/RPS6 co-clusters upon stimulation (n.s., $P>0.9999 ; n=21-24$ cells from 3 independent experiments). Data for Smn+/+; SMN2tgtg motoneurons are the same as those shown in Fig. 4b and included here to allow direct comparison. (c) In Smn-deficient motoneurons, number of RPL24/RPS6 coclusters colocalizing with ER does not increase upon stimulation (n.s., $P>0.9999 ; n=20-23$ cells from 3 independent experiments). Data for Smn+/+; SMN2tgtg motoneurons are the same as shown in Fig. $6 \mathrm{~b}$ are included here again to allow direct comparison. (d) Y10B and eEF2 antibodies were used to label ribosomes in the translation elongation phase and mCherry-ER was used to assess association of translating ribosomes with ER in growth cones of Smn-deficient motoneurons. White boxes indicate enlarged ROls within growth cones. (e) The number of Y10B/eEF2 co-clusters does not increase in Smn- 
deficient motoneurons upon stimulation (n.s., $P>0.9999 ; n=31-33$ cells from 3 independent experiments). Data for Smn+/+; SMN2tgtg motoneurons are the same as shown in Fig. 4d and included here again to allow direct comparison. (f) BDNF stimulation does not result in increased number of $\mathrm{Y} 10 \mathrm{~B} / \mathrm{eEF} 2$ co-clusters colocalizing with ER in Smn-deficient motoneurons (n.s., $\mathrm{P} \geq 0.6138 ; \mathrm{n}=31-33$ cells from 3 independent experiments). Data for $\mathrm{Smn}+/+$; SMN2tgtg motoneurons are the same as shown in Fig. $6 \mathrm{~d}$ and included here again to allow direct comparison. All data are normalized to no BDNF group of the corresponding genotype. Graphs are shown in scatter dot plot with mean \pm SEM. Statistical analyses: One-way ANOVA with Dunn's post-test.

\section{Supplementary Files}

This is a list of supplementary files associated with this preprint. Click to download.

- Fig.S1.tif

- Fig.S2.tif

- Video1.mp4

- Video2.mp4

- Video3.mp4

- Video4.mp4

- Video5.mp4

- Video6.mp4

- Video7.mp4

- Video8.mp4

- Video9.mp4 